ARTICLE

\title{
Molecular mechanism of the MORC4 ATPase activation
}

\author{
Adam H. Tencer', Khan L. Cox², Gregory M. Wright', Yi Zhang (1D 1, Christopher J. Petell ${ }^{3}$, Brianna J. Klein',

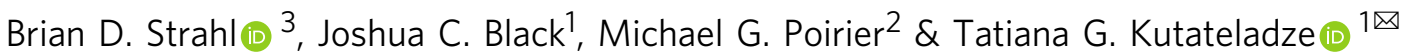

Human Microrchidia 4 (MORC4) is associated with acute and chronic pancreatitis, inflammatory disorders and cancer but it remains largely uncharacterized. Here, we describe the structure-function relationship of MORC4 and define the molecular mechanism for MORC4 activation. Enzymatic and binding assays reveal that MORC4 has ATPase activity, which is dependent on DNA-binding functions of both the ATPase domain and CW domain of MORC4. The crystal structure of the ATPaseCW cassette of MORC4 and mutagenesis studies show that the DNA-binding site and the histone/ATPase binding site of CW are located on the opposite sides of the domain. The ATPase and CW domains cooperate in binding of MORC4 to the nucleosome core particle (NCP), enhancing the DNA wrapping around the histone core and impeding binding of DNA-associated proteins, such as transcription factors, to the NCP. In cells, MORC4 mediates formation of nuclear bodies in the nucleus and has a role in the progression of S-phase of the cell cycle, and both these functions require $\mathrm{CW}$ and catalytic activity of MORC4. Our findings highlight the mechanism for MORC4 activation, which is distinctly different from the mechanisms of action observed in other MORC family members.

\footnotetext{
${ }^{1}$ Department of Pharmacology, University of Colorado School of Medicine, Aurora, CO 80045, USA. ${ }^{2}$ Department of Physics, Ohio State University, Columbus, OH 43210, USA. ${ }^{3}$ Department of Biochemistry \& Biophysics, the University of North Carolina School of Medicine, and UNC Lineberger Comprehensive Cancer Center, Chapel Hill, NC 27599, USA. ${ }^{凶}$ email: tatiana.kutateladze@cuanschutz.edu
} 
$\mathrm{M}$ icrorchidia 4 (MORC4) is a poorly characterized member of the new family of CW-type zinc finger nuclear proteins. MORC4 has been associated with acute and chronic pancreatitis and inflammatory bowel disorders, including Crohn's disease and ulcerative colitis, and more recently was found to be overly expressed in breast cancer cells and in diffuse large $\mathrm{B}$ cell lymphoma ${ }^{1-8}$. Analysis of breast cancer tissues shows that MORC4 is negatively regulated by microRNAs, including miR-193b-3p, and elevated MORC4 levels are linked to poor survival ${ }^{9}$. Despite growing evidence suggests an essential role of MORC4 in normal physiological and pathological processes, how this protein functions at molecular and cellular level is not well understood.

Members of the MORC family are characterized by their gyrase, Hsp90, histidine kinase, and MutL (GHKL)-type ATPase domain, which is also present in several chromatinmodifying enzymes ${ }^{10,11}$. Although the ability of MORC4 to hydrolyze ATP has not been investigated, the homologous MORC proteins, such as MORC2 and MORC3 are enzymatically active. MORC3 has been shown to exist in an autoinhibited state and is activated through binding to methylated histone mark $\mathrm{H} 3 \mathrm{~K} 4 \mathrm{me} 3$, associated with transcriptionally active chromatin ${ }^{12-16}$. Conversely, MORC2 does not recognize H3K4me3 and is necessary for the human silencing hub (HUSH)-dependent silencing of transgenes integrated at chromatin loci with the methylated histone mark $\mathrm{H} 3 \mathrm{~K} 9 \mathrm{me} 3^{17,18}$. The ATPase domain of MORCs is followed by a CW-type zinc finger and one or more coiled-coil regions (Fig. 1a). Recognition of $\mathrm{H} 3 \mathrm{~K} 4 \mathrm{me} 3$ by MORC3 is mediated by the CW domain, and this interaction regulates the catalytic activity of MORC3 and is required for the MORC 3 recruitment to chromatin and formation of liquid-liquid phase separation (LLPS) droplets in the nucleus $13,15,19,20$. While we have begun understanding the markedly different mechanisms of action and some biological roles of the individual MORC2 and MORC3 proteins, virtually nothing is known about the function of MORC4.

Here, we describe the unique molecular mechanism for MORC4 activation. We show that MORC4 is an ATPase with an intrinsic DNA-dependent but histone-independent enzymatic activity. The ATPase and $\mathrm{CW}$ domains of MORC4 cooperate in binding of MORC4 to the NCP, impeding the association of transcription factors with the NCP. Our cell data reveal two biological functions of MORC4: it regulates the formation of nuclear bodies (NBs) in the nucleus and plays a role in the cell cycle S-phase progression with the ATPase activity and chromatin binding being necessary for both functions.

\section{Results}

MORC4 is an ATPase. To determine whether MORC4 is catalytically active, we produced the ATPase domain of human MORC4 and examined its ability to hydrolyze ATP in enzymatic assays (Fig. 1a-c). Following incubation of the ATPase domain with ATP, 2-amino-6-mercapto-7-methylpurine ribonucleoside and nucleoside phosphorylase, the release of inorganic phosphate generated by the hydrolysis of ATP to ADP was monitored by measuring an increase in absorbance at $360 \mathrm{~nm}$. We found that the MORC4 ATPase domain itself possesses little ATP hydrolyzing activity, however addition of 147 base pair (bp) 601 Widom DNA at a 1:1 molar ratio led to a $\sim 4$-fold increase in the rate of ATP hydrolysis, indicating that the catalytic activity of the ATPase domain is DNA-dependent (Fig. 1b). A similar increase in the ATP hydrolysis rate was observed upon adding 601 DNA to the ATPase domain linked to the CW domain of MORC4 in the ATPaseCW cassette (Fig. 1a, c). Neither methylated histone peptide H3K4me3 nor unmodified $\mathrm{H} 3$ peptide (both aa 1-12 of a
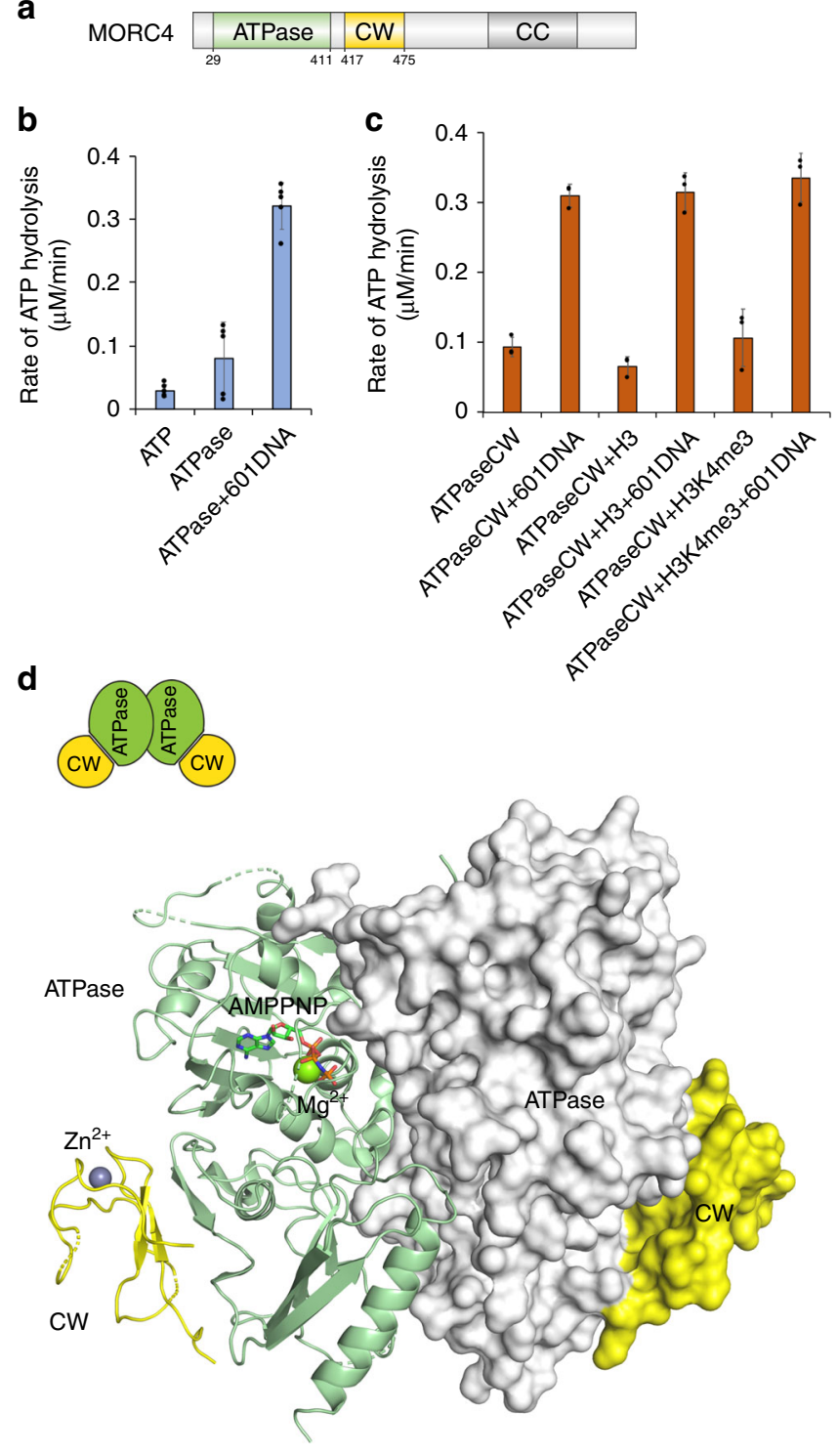

MORC4 ATPaseCW dimer

Fig. 1 MORC4 is an ATPase. a MORC4 domain architecture. The ATPase and $\mathrm{CW}$ domains are colored green and yellow, respectively. The ATPaseCW cassette construct contains aa 29-486 of MORC4 (Q8TE76). b, c Rates of ATP hydrolysis by the ATPase domain of MORC4 (b) and the ATPase-CW cassette of MORC4 (c). Data are represented as mean values $+/-$ S.D. of at least three independent experiments $(n \geq 3)$. Source data are provided in a Source Data file. d The crystal structure of the dimeric MORC4 ATPase-CW/AMPPNP complex. In protomer A, the ATPase domain and the $\mathrm{CW}$ domain are shown in a ribbon diagram and colored green and yellow, respectively. In protomer $B$, the surface representation of the ATPase domain (white) and the CW domain (yellow) is shown. The magnesium (green) and zinc (gray) atoms are shown as spheres. The AMPPNP molecule is in stick representation. Dashed lines represent residues and loops that are not defined by electron density.

H3) stimulated a further increase in the rate of ATP hydrolysis, implying that the enzymatic activity is largely histone independent (we discuss binding of $\mathrm{CW}$ to $\mathrm{H} 3$ below). The nucleosome core particle (NCP) also stimulated the catalytic activity of the ATPaseCW cassette (Supplementary Fig. 1). Together, these data demonstrate that the ATPase activity is a function of MORC4, which requires the presence of DNA. 
Structure of the MORC4 ATPaseCW cassette. To gain mechanistic insight into the MORC4 activation process, we crystallized ATPaseCW (aa 29-486 of human MORC4) in complex with the non-hydrolyzable ATP analog, adenylylimidodiphosphate (AMPPNP), collected and analyzed X-ray crystallographic data, and refined the structure to 2.9 A resolution (Fig. 1d and Supplementary Table 1). The structure reveals a symmetric parallel homodimer with each ATPaseCW protomer coordinating one molecule of AMPPNP and one $\mathrm{Mg}^{2+}$ ion. The ATPase module folds into the GHKL-type domain, consisting of a mixture of $\alpha$-helices and $\beta$-strands, and is involved in the formation of a large dimer interface, which was also observed in the respective structures of MORC3 and MORC2 ${ }^{14,15,17}$. Two zincbinding CW domains tightly pack against the ATPase domains and are positioned on the sides of the ATPase domains opposite to the dimer interface and are far apart (colored yellow in Fig. 1d).

Each CW domain interacts mainly with the loops between $\alpha 8$ and $\beta 10$ of the ATPase domain (Supplementary Fig. 2). The residues P279, K289, and $\mathrm{K} 297$ of the ATPase domain form a number of polar and electrostatic contacts with the Q424, W426, Q428, D430, W435, and A449 residues of the CW domain. In addition, the hydrophobic side chain of M294 is caged between the aromatic rings of W426 and W435 of CW, and the side chain of R69 of the ATPase domain is restrained by hydrogen bonds with the backbone carbonyl groups of D430 and E431 and the carboxyl group of E431 of CW.

Strikingly, while the MORC4 ATPaseCW cassette structure superimposes well with the structure of ATPaseCW in the homologous protein MORC $3^{15}$ (RMSD of $1.5 \AA$ ) and shares high sequence similarity with MORC3 (Supplementary Fig. 3), the mechanisms of action of the two proteins differ. The ATPaseCW cassette of MORC4 is capable of binding to DNA and therefore is active, whereas the ATPaseCW cassette of MORC3 represents an autoinhibited state, which does not inherently bind DNA. Binding of the CW domain of MORC3 to histone H3K4me3, which leads to the disruption of the CW:ATPase complex ${ }^{14}$ (the same surface of CW is involved in binding to the ATPase domain and $\mathrm{H} 3 \mathrm{~K} 4 \mathrm{me} 3^{13-15}$ ), is required for the release of autoinhibition, thus allowing interaction with DNA and resulting in MORC3 activation $^{13,15}$. Why is the catalytic activity of MORC4 independent of histone binding in contrast to the MORC3 catalytic activity and what does then stimulate MORC4 activation? To explain this, we considered three possibilities, i.e., MORC4 does not bind histone tails, DNA binding occludes the histone-binding site, or DNA binding promotes the catalytic activity of MORC4 independently of histone binding.

The CW domain of MORC4 selects for histone H3K4me3. A set of zinc fingers, including the $\mathrm{CW}$ domain of $\mathrm{MORC}^{13}$, has been shown to recognize histone sequences, therefore we tested whether the MORC4 CW domain either isolated (in this section) or linked to the ATPase domain in the ATPaseCW cassette (below) is capable of binding to histone tails using highthroughput histone peptide microarrays, pull-down assays and NMR (Fig. 2). In microarrays, GST-tagged CW was incubated with a library of over $\sim 250$ synthetic histone peptides containing single posttranslational modifications (PTMs) or combinations of PTMs, including acetylated and methylated lysine residues, phosphorylated serine and threonine residues and methylated arginine residues known to be present in the core and variant histone proteins (Supplementary Data 1). We found that the MORC4 CW domain binds to the N-terminal histone $\mathrm{H} 3$ peptides, selecting for methylated $\mathrm{H} 3 \mathrm{~K} 4$ sequences and does not recognize histone $\mathrm{H} 4, \mathrm{H} 2 \mathrm{~A}$, and $\mathrm{H} 2 \mathrm{~B}$ (Fig. 2a, b). These results were corroborated by pull-down assays, in which GST-tagged CW domain of MORC4 was incubated with biotinylated unmodified and modified histone $\mathrm{H} 3$ peptides. As shown in Fig. 2c, $\mathrm{CW}$ associated with methylated $\mathrm{H} 3 \mathrm{~K} 4$ peptides, preferring the higher (tri)methylation state, H3K4me3. While methylation of the neighboring sites H3R2 and H3R8 appeared to not affect binding of CW to H3K4me3, phosphorylation of H3T3 or H3T6 disrupted interaction with the doubly modified H3T3phK4me3 and $\mathrm{H} 3 \mathrm{~K} 4 \mathrm{me} 3 \mathrm{~T} 6 \mathrm{ph}$ peptides.

To further compare binding of the MORC4 CW domain to methylated and unmodified $\mathrm{H} 3 \mathrm{~K} 4$, we produced ${ }^{15} \mathrm{~N}$-labeled $\mathrm{CW}$ and examined it in ${ }^{1} \mathrm{H},{ }^{15} \mathrm{~N}$ heteronuclear single quantum coherence (HSQC) experiments (Fig. 2d, e). Titration of the $\mathrm{H} 3 \mathrm{~K} 4 \mathrm{me} 3$ peptide (aa $1-12$ of $\mathrm{H} 3$ ) resulted in substantial chemical shift perturbations (CSPs) in the MORC4 CW domain, indicating direct interaction. Throughout the gradual addition of the peptide, a number of crosspeaks corresponding to the free state of the protein disappeared, and simultaneously, another set of resonances, corresponding to the bound state, appeared. This pattern of CSPs indicates a tight binding in the slow exchange regime on the NMR time scale. However, interaction of the MORC4 CW domain with the unmodified $\mathrm{H} 3$ peptide was weaker judging by CSPs in the intermediate exchange regime. In support, dissociation constants $\left(K_{\mathrm{d}} \mathrm{s}\right)$ for the complexes of the MORC4 CW domain with the H3K4me3 and H3K4 peptides were found to be $\sim 0.1$ and $2.7 \mu \mathrm{M}$, respectively, as measured by tryptophan fluorescence (Fig. 2f, g and Supplementary Fig. 4). These results demonstrate that the MORC4 CW domain is not only a selective reader of $\mathrm{H} 3 \mathrm{~K} 4 \mathrm{me} 3$ but also one of the strongest effectors of H3K4me3, which exhibits 7-10 fold higher binding affinity to this PTM than other readers ${ }^{21,22}$.

Furthermore, consecutive NMR titration experiments showed that the two ligands of the MORC4 CW domain-the ATPase domain and histone $\mathrm{H} 3$-compete for binding to CW. Gradual addition of the isolated unlabeled ATPase domain to the ${ }^{15} \mathrm{~N}$ labeled MORC4 CW domain led to a decrease in intensities of amide resonances, which suggested formation of the large ATPase:CW complex (Fig. 3a). Titration of the ATPase domain into the CW:H3 complex caused CSPs that were indicative of the disruption of the CW:H3 complex and formation of the complex between $\mathrm{CW}$ and the ATPase domain (Fig. 3b). We concluded that MORC4 CW is capable of binding to either $\mathrm{H} 3$ or the ATPase domain and that, similarly to MORC $3^{13-15}$, the ATPasebinding site and the histone-binding site of CW overlap.

The MORC4 CW domain binds DNA. Considering the ability of the MORC4 ATPaseCW cassette to be activated by DNA, we investigated whether the $\mathrm{CW}$ domain contributes to the DNA binding by testing it in electrophoretic mobility shift assay (EMSA) (Fig. 3c). 601 DNA was incubated with increasing concentrations of $\mathrm{CW}$, and the reaction mixtures were resolved on a $5 \%$ native polyacrylamide gel. A gradual increase in the amount of added CW resulted in the shift and disappearance of the free 601 DNA band and appearance of a band smear corresponding to the complex of CW with $601 \mathrm{DNA}$, indicating the direct interaction (Fig. 3c, e). Unlike the CW domain of MORC4, the CW domain of homologous MORC3 was largely incapable of binding to 601 DNA, as no DNA band shift was observed in EMSA with MORC3 CW (Fig. 3d).

To identify the DNA-binding site of the MORC4 CW domain, we examined its electrostatic surface potential (ESP) and compared it to that of the MORC3 CW domain (Fig. 3f, g). Analysis of ESP of MORC4 CW revealed that particularly one side of $\mathrm{CW}$, which is opposite to the H3K4me3- and ATPasebinding sites, is highly positively charged. Specifically, K460, 


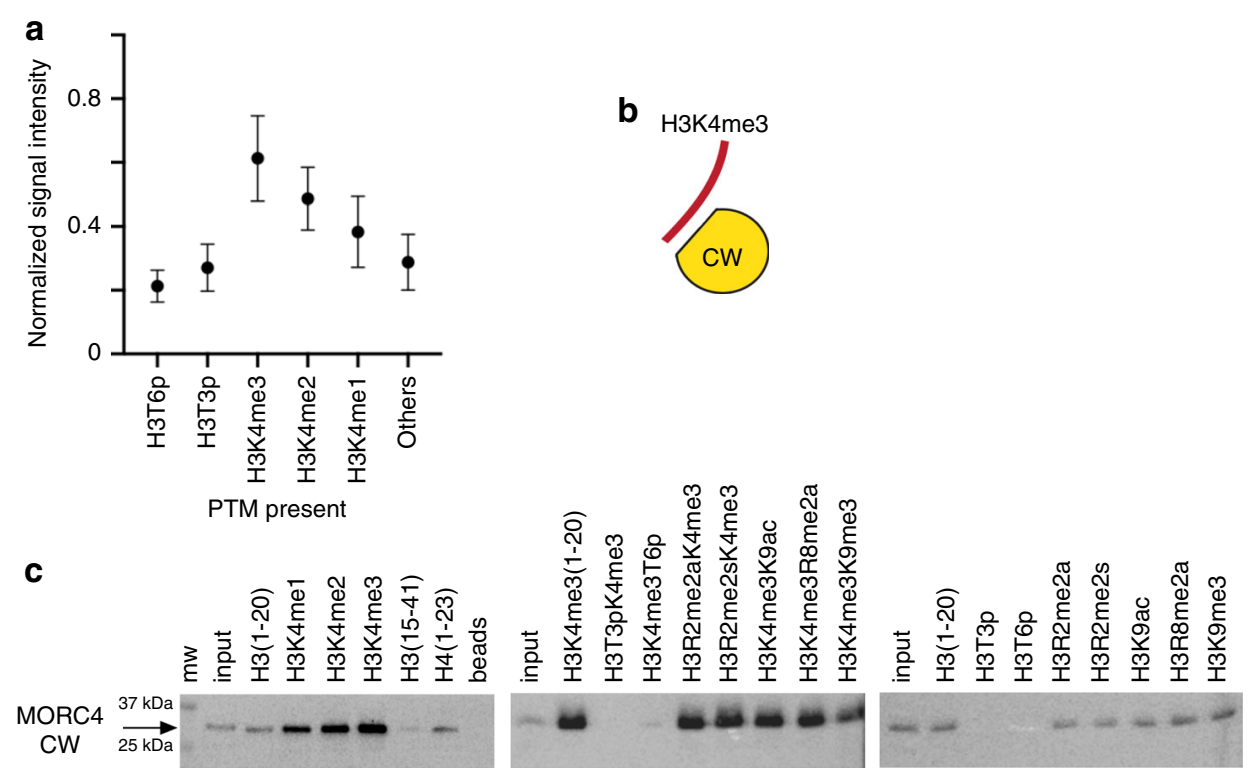

d

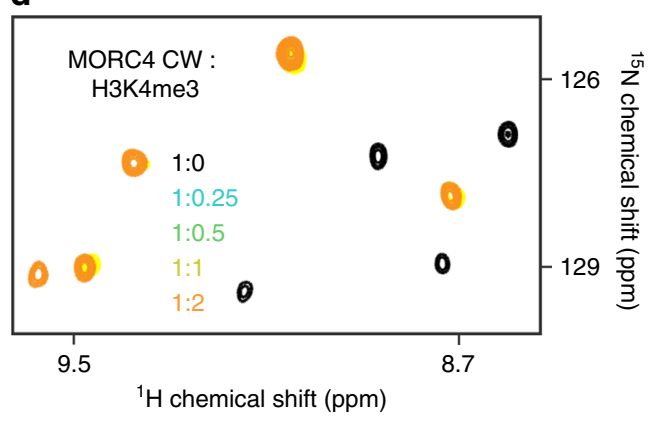

e

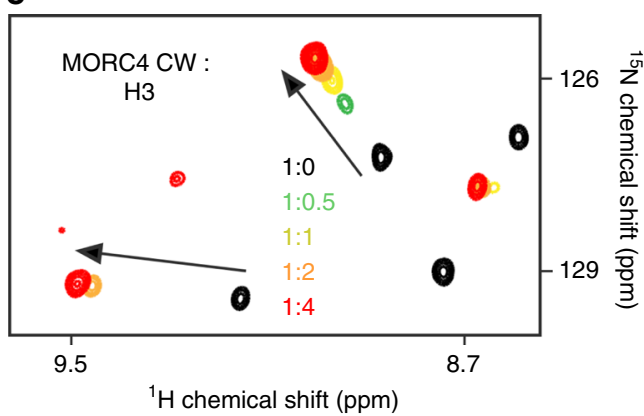

f

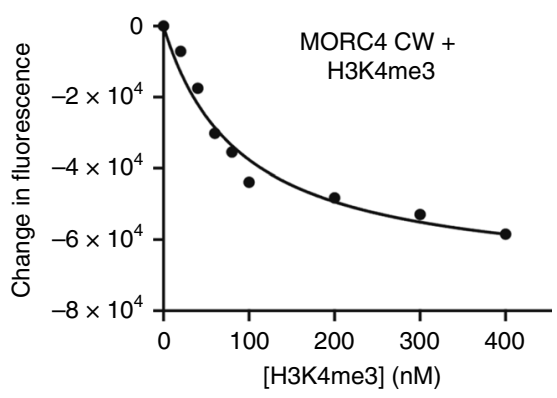

g

Binding affinities of MORC4 $\mathrm{CW}$

\begin{tabular}{|lc|}
\hline peptide & $K_{\mathrm{d}}(\mu \mathrm{M})$ \\
\hline H3K4me3 & $0.112 \pm 0.019$ \\
$\mathrm{H} 3$ & $2.7 \pm 0.4$ \\
\hline
\end{tabular}

Fig. 2 The CW domain of MORC4 recognizes H3K4me3. a Normalized average microarray signal intensities detected upon binding of the GST-tagged MORC4 CW domain to the peptides carrying indicated PTMs. Data are represented as mean values $+/-$ S.D. from $n \geq 3$ independent arrays. See also Supplementary Data 1. b Cartoon representation of CW in complex with histone H3K4me3 tail. c Peptide pull-down assays of the GST-tagged MORC4 CW domain using the indicated histone H3 peptides. Images are representative of three independent experiments with similar results ( $n=3$ ). Source data are provided in a Source Data file. d, e Superimposed ${ }^{1} \mathrm{H},{ }^{15} \mathrm{~N} \mathrm{HSQC}$ spectra of the ${ }^{15} \mathrm{~N}$-labeled WT MORC4 CW domain collected upon titration with the histone H3K4me3 (d) and unmodified H3 (e) peptides. Spectra are color coded according to the protein-to-peptide molar ratio. $\mathbf{f}$ Representative binding curve used to determine $K_{d}$ values by tryptophan fluorescence. $\mathbf{g}$ Binding affinities of MORC4 $\mathrm{CW}$ for the indicated histone $\mathrm{H} 3$ peptides measured by tryptophan fluorescence. Data are represented as mean values $+/-$ S.D. from three independent experiments $(n=3)$. Source data are provided in a Source Data file.

R462 and R463 form a well-defined positively charged cluster on the protein surface, which suggested to us that these residues might be involved in binding to the negatively charged DNA. We generated the triple mutant of the MORC4 CW domain, $\mathrm{K} 460 \mathrm{~A} / \mathrm{R} 462 \mathrm{~A} / \mathrm{R} 463 \mathrm{~A}\left(\mathrm{CW}_{\mathrm{KRR}}\right)$, and tested its interaction with 601 DNA by EMSA. We did not observe the 601 DNA band shift even at the DNA to $\mathrm{CW}_{\mathrm{KRR}}$ molar ratio of 1 to 200 (Fig. 3h), whereas H3K4me3 binding activity of this mutant appeared to be unaffected based on the pattern of CSPs seeing in NMR titration experiment with the H3K4me3 peptide (Fig. 3i). Collectively, these data suggest that the K460, R462, and R463 residues of the CW domain of MORC4 are required for the strong interaction with DNA and that the DNA-binding site and the histone/ATPase-binding sites are located on the opposed sides of the MORC4 CW domain. 


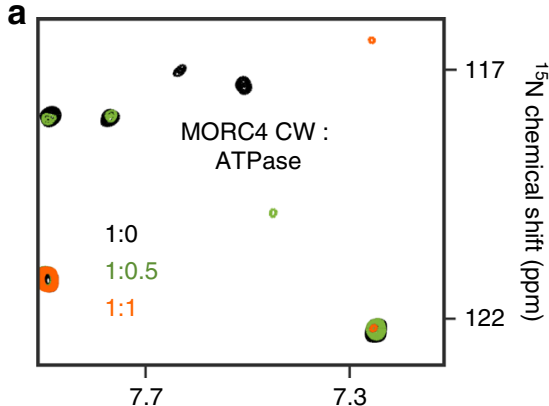

${ }^{1} \mathrm{H}$ chemical shift $(\mathrm{ppm})$

C

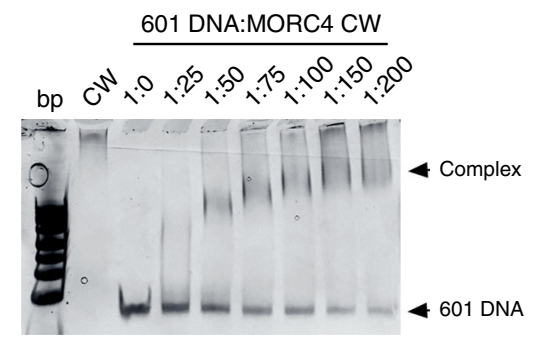

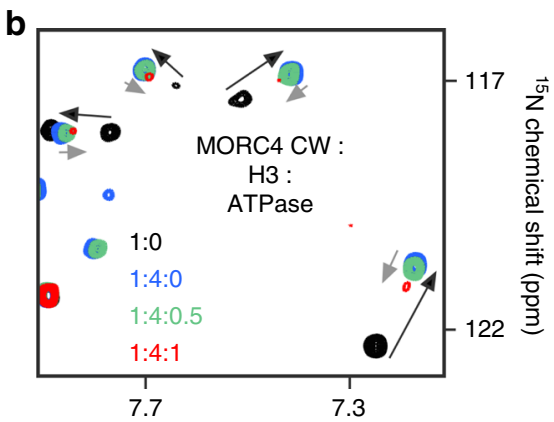

${ }^{1} \mathrm{H}$ chemical shift (ppm)

d

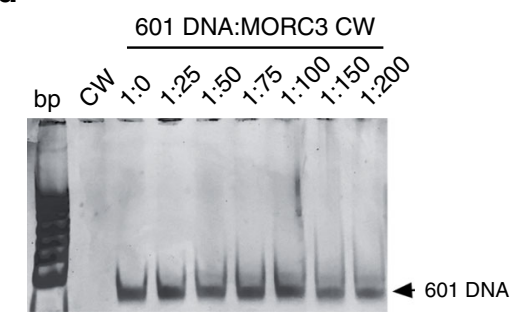

e

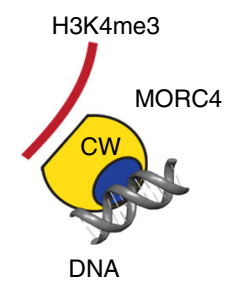

h

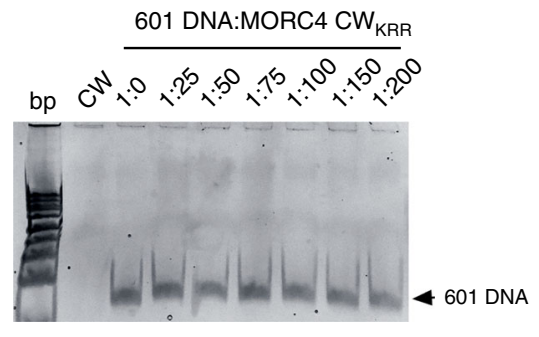

f

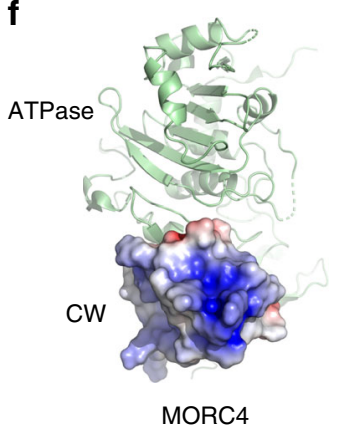

MRC4

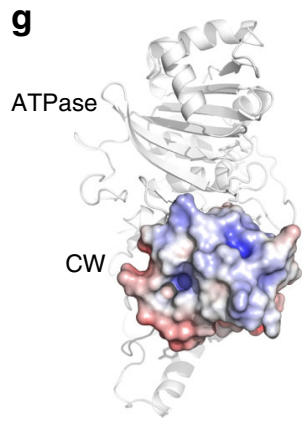

MORC3

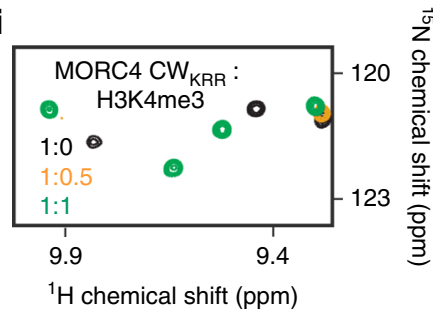

Fig. 3 MORC4 CW binds to DNA. a Superimposed ${ }^{1} \mathrm{H},{ }^{15} \mathrm{~N}$ HSQC spectra of the ${ }^{15} \mathrm{~N}$-labeled MORC4 $\mathrm{CW}$ domain collected upon titration with the unlabeled ATPase domain of MORC4. b Superimposed ${ }^{1} \mathrm{H},{ }^{15} \mathrm{~N}$ HSQC spectra of the ${ }^{15} \mathrm{~N}$-labeled MORC4 $\mathrm{CW}$ domain (in complex with $\mathrm{H} 3$ peptide) collected upon titration with the unlabeled ATPase domain. Spectra in $(\mathbf{a}, \mathbf{b})$ are color coded according to the protein-to-ligands molar ratio. (c, d) EMSA with 601 DNA in the presence of increasing amounts of MORC4 CW (c) and MORC3 CW (d). e Cartoon representation of MORC4 CW in complex with histone H3K4me3 tail and DNA. f, $\mathbf{g}$ Electrostatic surface potential of the CW domain of MORC4 (f) and MORC3 (g) (within the ATPase-CW cassette) was generated using APBS in Pymol with a range of $-5 / 5 \mathrm{kT} / \mathrm{e}$ and colored blue and red for positive and negative charges, respectively. The ATPase domain is shown as ribbon and colored green and white in MORC4 and MORC3, respectively. The missing loops (residues K441 and R463) in the model of MORC4 CW were completed for this figure. $\mathbf{h}$ EMSA with 601 DNA in the presence of increasing amounts of mutant MORC4 CW KRR. $\mathbf{i}$ Superimposed ${ }^{1} \mathrm{H},{ }_{15}^{15} \mathrm{~N} \mathrm{HSQC}$ spectra of the ${ }^{15} \mathrm{~N}$-labeled MORC4 $\mathrm{CW}_{\mathrm{KRR}}$ domain collected upon titration with the histone H3K4me3 peptide. Spectra are color coded according to the protein-to-peptide molar ratio.

DNA-binding activity of the MORC4 CW domain is essential. Can binding of the ATPaseCW cassette to DNA occlude the histone-binding site? We tested this possibility by measuring binding affinities of the ATPaseCW cassette for the $\mathrm{H} 3$ peptides in the absence and presence of 601 DNA by tryptophan fluorescence (Fig. $4 \mathrm{a}-\mathrm{c}$ ). We found that the ATPaseCW cassette binds to H3K4me3 only slightly (and not significantly) weaker as compared to binding of the isolated $\mathrm{CW}$ domain to the same $\mathrm{H} 3 \mathrm{~K} 4 \mathrm{me} 3$ peptide ( $K_{\mathrm{d}} \mathrm{s}$ of $180 \mathrm{nM}$ and $112 \mathrm{nM}$, respectively) (Figs. 2f, g and 4a, c and Supplementary Fig. 4). However, interaction of the ATPaseCW:601 DNA complex with H3K4me3 peptide was 4.5 -fold stronger $\left(K_{\mathrm{d}}\right.$ of $\left.40 \mathrm{nM}\right)$, implying that DNA binding enhances rather than impedes binding to $\mathrm{H} 3 \mathrm{~K} 4 \mathrm{me} 3$ (Fig. 4d). These results led us to the 


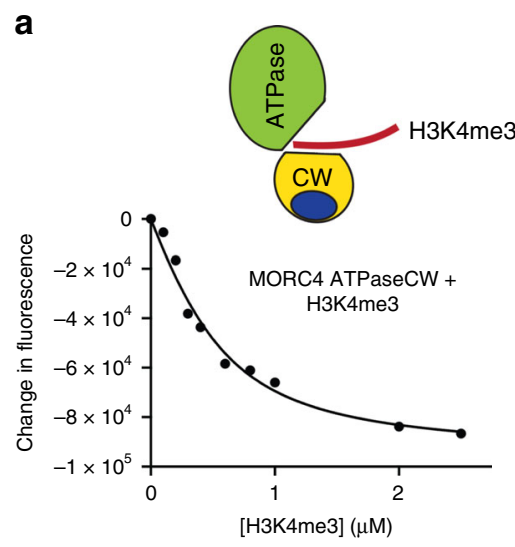

C

\begin{tabular}{|lcc|}
\multicolumn{3}{l}{ Binding affinities of MORC4 ATPaseCW } \\
\hline Peptide & Protein:601 DNA & $K_{\mathrm{d}}(\mu \mathrm{M})$ \\
\hline H3K4me3 & $1: 0$ & $0.18 \pm 0.08$ \\
H3K4me3 & $1: 1$ & $0.040 \pm 0.009$ \\
H3 & $1: 0$ & $12 \pm 4$ \\
\hline
\end{tabular}

e

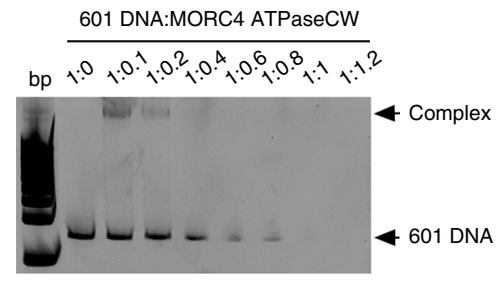

$\mathbf{9}$

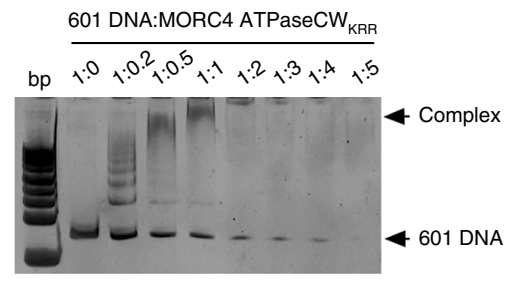

i

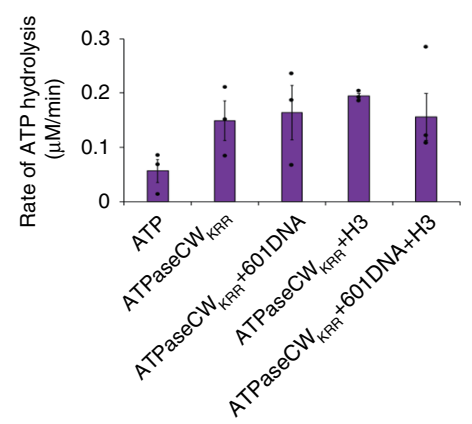

current model for the MORC4 activation, in which DNA binding by the ATPaseCW cassette (both ATPase and CW domains are involved in this interaction) promotes the catalytic activity and binding of CW to H3K4me3 (Fig. 4d). This model was further corroborated by EMSA experiments (Fig. 4e-h). First, the presence of $\mathrm{H} 3 \mathrm{~K} 4 \mathrm{me} 3$ peptide slightly increased binding of either wild-type ATPaseCW or mutated

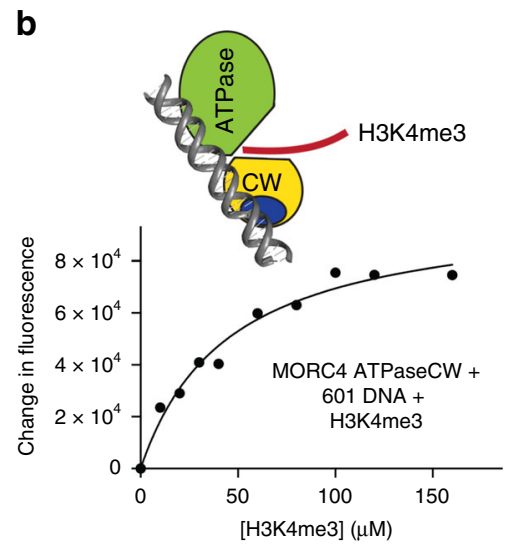

d

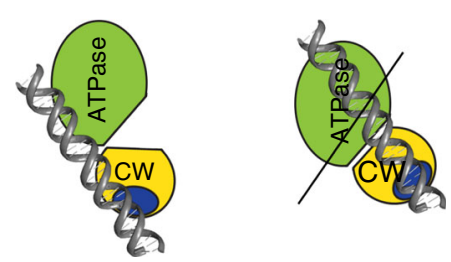

f

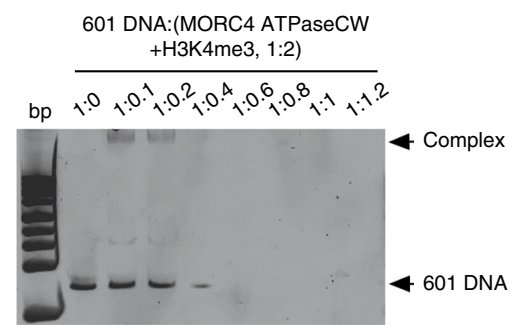

$\mathbf{h}$

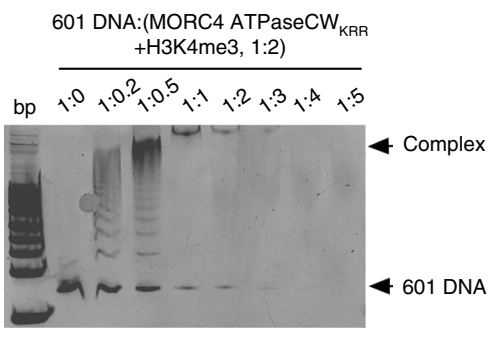

j

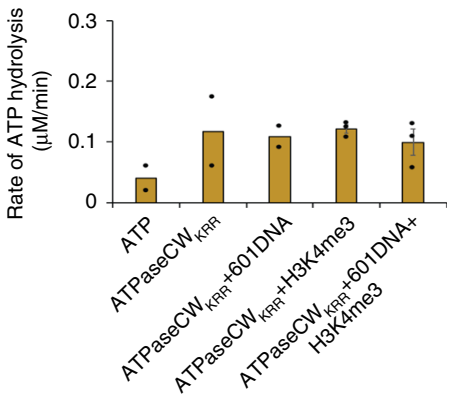

ATPaseCW $_{\mathrm{KRR}}$ to $601 \mathrm{DNA}$, and second, the DNA-binding function of the mutated $\mathrm{ATPaseCW}_{\mathrm{KRR}}$ cassette was notably reduced, demonstrating the role of $\mathrm{CW}$ in the association with DNA. The importance of the DNA-binding activity of CW in activation of MORC4 was underscored by the fact that the mutant ATPaseCW $\mathrm{KRR}_{\mathrm{K}}$ cassette failed to be stimulated to hydrolyze ATP in enzymatic assays (Fig. 4i, j). 
Fig. 4 DNA binding by MORC4 CW is necessary for ATP hydrolysis. a, b Representative binding curves used to determine $K_{d}$ values by fluorescence. Cartoon representations of MORC4 ATPaseCW binding to histone $\mathrm{H} 3$ tail in the absence (a) and presence (b) of DNA are shown above the curves. c Binding affinities of MORC4 ATPaseCW for the indicated histone H3 peptides (with or without 601 DNA) measured by tryptophan fluorescence. Data are represented as mean values +/-S.D. from three independent experiments $(n=3)$. Source data are provided in a Source Data file. d Models of the MORC4 ATPaseCW-DNA complex. e-h EMSA with 601 DNA in the presence of increasing amounts of WT (e, $\mathbf{f})$ and mutant (g, $\mathbf{h})$ MORC4 ATPaseCW in the absence $(\mathbf{e}, \mathbf{g})$ and presence $(\mathbf{f}, \mathbf{h})$ of histone $\mathrm{H} 3 \mathrm{~K} 4 \mathrm{me} 3$ peptide. Experiments in (e) and ( $\mathbf{g})$ were repeated ten times and two times, respectively.

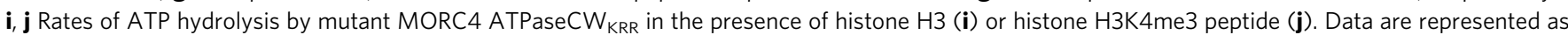
mean values +/-S.D. of three independent experiments. Error was not calculated for the first three data sets containing two data points in $(\mathbf{j})(n=2)$. Source data are provided in a Source Data file.

Extensive DNA-binding site of the MORC4 ATPaseCW cassette. To delineate the DNA-binding site of the ATPaseCW cassette, we mutated four positively charged surface clusters in the ATPase domain, producing the K213Q/K214Q, R225Q/ K227Q/K314Q, K352Q/R355Q, and K401Q/K403Q mutants of ATPaseCW, and tested binding of these mutants to 601 DNA by EMSA (Fig. 5). Among the mutants, the R225Q/K227Q/K314Q mutant (Fig. 5b), and to a lesser degree the K401Q/K403Q mutant (Fig. 5c), showed the most evident reduction in DNAbinding activity, whereas the reduction in DNA binding was less pronounced for other two mutants, K352Q/R355Q and K213Q/ K214Q (Fig. 5d, e). The K460/R462/R463 cluster in the CW domain (Fig. 5a, blue oval) and the R225/K227/K314 and K401/ K403 clusters in the ATPase domain (Fig. 5a, orange and light blue ovals) are separated by over $40 \AA$, indicating that a large surface of the ATPaseCW cassette is involved in binding to DNA. Furthermore, the R225/K227/K314 cluster is formed by R225 and $\mathrm{K} 227$ from one protomer and K314 from another protomer in the dimeric structure of ATPaseCW, suggesting that dimerization might be important for the association with DNA. Indeed, EMSA experiments showed that stimulation of ATPaseCW dimerization through adding AMPPNP increases binding to 601 DNA (Supplementary Figs. 5 and 6 ) and that at least $\sim 30$ bp DNA is needed for the ATPaseCW cassette to appreciably interact with DNA (Supplementary Fig. 7).

MORC4 impedes binding of DNA-associated proteins. To gain insight into the MORC4 activity at chromatin, we investigated the consequences of the association of the MORC4 ATPaseCW cassette with NCP, a fundamental unit of chromatin. Both the ATPase domain alone and the ATPaseCW cassette of MORC4 bound to $\mathrm{NCP}_{147}$ (the nucleosome reconstituted with the $147 \mathrm{bp}$ 601 nucleosome positioning DNA sequence) in fluorescence polarization assays $\left(S_{1 / 2}=2.3 \mu \mathrm{M}\right.$ and $0.4 \mu \mathrm{M}$, respectively) and EMSA (Supplementary Figs. 8 and 9). However, interaction of the ATPase domain and the ATPaseCW cassette with $\mathrm{NCP}_{207}$ (the nucleosome reconstituted with $207 \mathrm{bp}$ DNA containing the 601 sequence flanked with a $30 \mathrm{bp}$ linker on either side and internally labeled with fluorescein $27 \mathrm{bp}$ in from the 5' end) was increased $\sim 5$-fold and $\sim 2$-fold, respectively (Fig. 6a). These results suggest that extra-nucleosomal DNA enhances binding of MORC4. Importantly, binding of ATPaseCW to either $\mathrm{NCP}_{147}$ or $\mathrm{NCP}_{207}$ was $\sim 2.5-6$ fold tighter than the binding of the ATPase domain only, pointing to a contribution from the $\mathrm{CW}$ domain to the interaction with NCPs.

The impact of this interaction on the nucleosome dymnamics and unwrapping-wrapping equilibrium was investigated by Förster resonance energy transfer $(\mathrm{FRET})^{23}$. We designed and prepared $\mathrm{NCP}_{252}$ using $252 \mathrm{bp}$ DNA, which contained the 601 sequence with a $30 \mathrm{bp}$ linker at the $5^{\prime}$ end, a $75 \mathrm{bp}$ linker at the $3^{\prime}$ end, and the transcription factor Gal4 DNA-binding site replacing bases 8-26 in the 601 sequence (Fig. 6b). The Cy3 donor fluorophore was attached to the $5^{\prime}$ end of the 601 DNA adjacent to the Gal4-binding site, and the Cy5 acceptor fluorophore was attached to histone H2A(K119C). This placed $\mathrm{Cy} 3$ in the proximity of one of the Cy5 fluorophores, therefore a significant FRET signal is expected from a fully wrapped nucleosome, while a reduced FRET is expected when the $\mathrm{NCP}_{252}$ is in a more open, partially unwrapped state (Fig. 6c). Titration of the Gal4 DNA-binding domain into Cy3-Cy5 labeled $\mathrm{NCP}_{252}$ led to a decrease in FRET due to Gal4 binding to its target site and stabilization of the unwrapped state (Fig. 6d). Consistent with previous measurements ${ }^{24}$, the value for the Gal4 concentration at which FRET efficiency is reduced by $50 \%\left(\mathrm{~S}_{1 / 2}\right)$ was found to be $20 \mathrm{nM}$.

To determine the effect of MORC4 on the nucleosome unwrapping-wrapping equilibrium, we next titrated Gal4 into $\mathrm{NCP}_{252}$ in the presence of ATPaseCW and ATP (Fig. 6e). The Gal4 ability to bind $\mathrm{NCP}_{252}$ was decreased $\sim 5$-fold, resulting in a $S_{1 / 2}=100 \mathrm{nM}$ (Fig. 6e). The presence of ATP was essential, as only 2 -fold decrease was observed in the absence of ATP $\left(S_{1 / 2}=40 \mathrm{nM}\right)$. Similar results were obtained in FRET experiments using $\mathrm{Cy} 3-\mathrm{Cy} 5$ labeled $\mathrm{NCP}_{147}$ containing the targeting site of another transcription factor, LexA, at bases 8-27 in the 601 DNA sequence (Fig. 5f). Addition of MORC4 ATPaseCW to the reaction resulted in a decrease in binding of LexA to $\mathrm{NCP}_{147}$ due to the stabilization of the wrapped state of $\mathrm{NCP}_{147}$. Collectively, these data reveal that binding of the MORC4 ATPaseCW cassette to the nucleosome shifts the unwrapping-wrapping equilibrium toward the wrapped state, enhancing the nucleosome stability and impeding binding of DNA-binding proteins, such as transcription factors and coactivators.

ATPase activity of MORC4 regulates formation of NBs. Our findings demonstrate that the ATPaseCW cassette of MORC4 is capable of engaging chromatin. To determine the importance of this region in MORC4 functioning in vivo, we assessed the subcellular localization of MORC4 in HEK293T cells. Cells were transfected with mCherry-tagged wild-type and mutated fulllength MORC4, and $48 \mathrm{~h}$ post transfection mCherry-tagged proteins were visualized in live cells by confocal fluorescence microscopy (Fig. 7a and Supplementary Fig. 10). We found that wild-type mCherry-MORC4 localizes to the nucleus, forming large NBs with the average size of $0.52 \mu \mathrm{m}^{2}$. In 124 cells quantified, the number of MORC4 NBs ranges from 1 to 18 with an average of 4.93 per cell (Fig. 7b). The E56A mutant of MORC4 that retains its DNA-binding activity (Fig. 5f) but has catalytically impaired ATPase domain (Supplementary Fig. 11) showed a notable increase in the number of NBs and a decrease in the size of NBs in 127 cells quantified, indicating that the catalytic activity of MORC4 regulates the formation of NBs (Fig. 7a). In contrast, MORC4 W435A mutant, in which the structure of the CW domain is disrupted (Supplementary Fig. 12), was dispersed throughout the cell, revealing the critical role of $\mathrm{CW}$ in nuclear localization of MORC4 and its association with chromatin in vivo (Fig. 7a). 


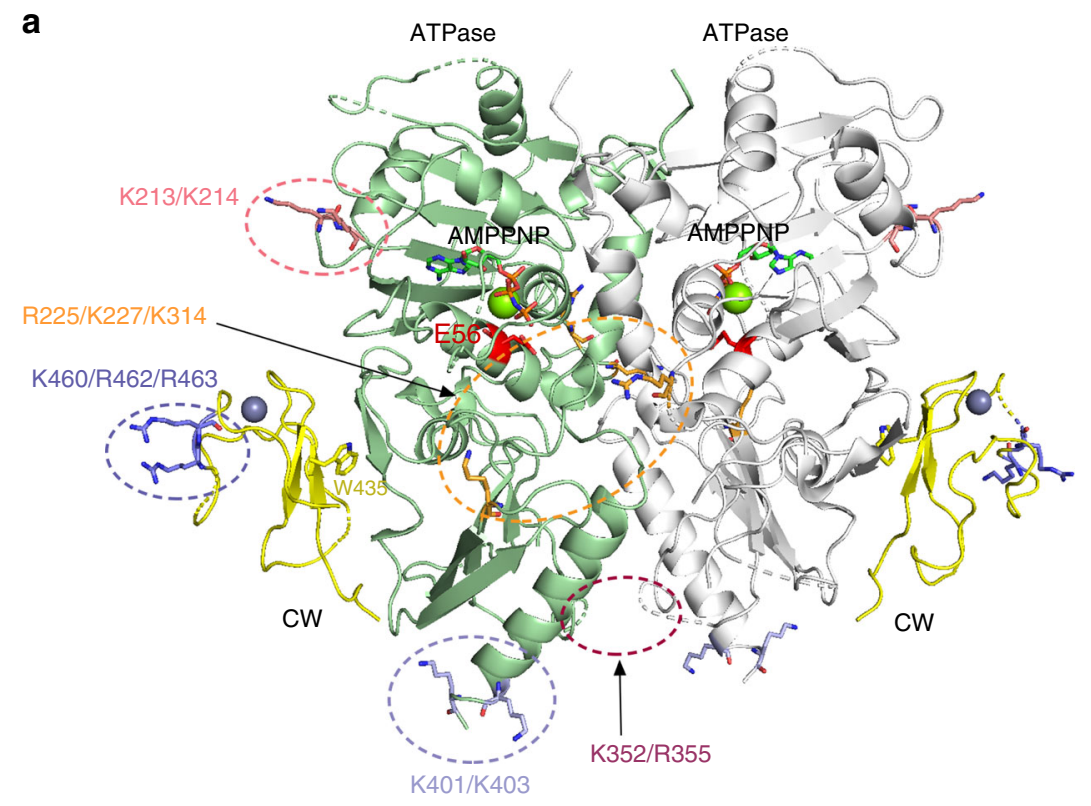

MORC4 ATPaseCW dimer

b

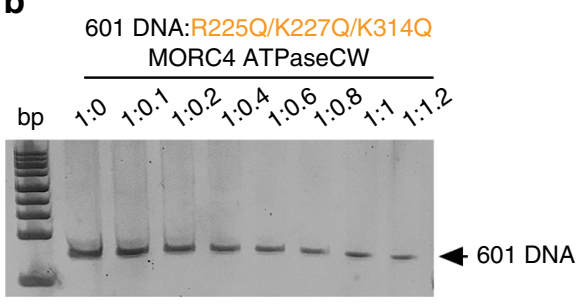

d

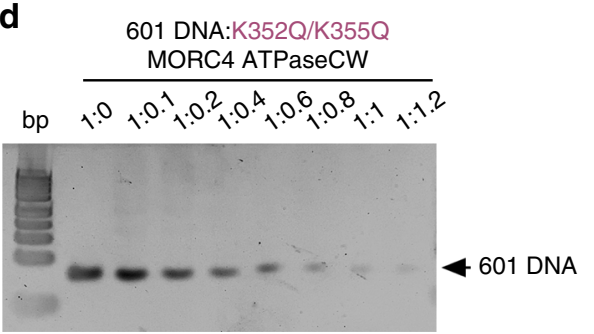

C
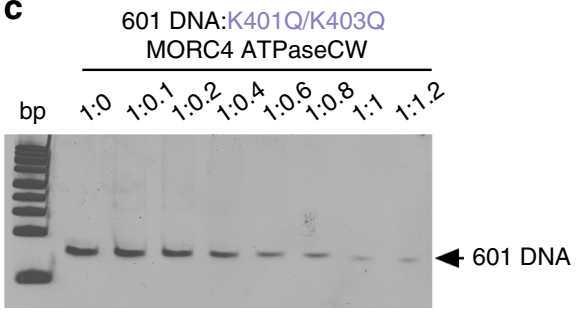

e

f 601 DNA:E56A MORC4 ATPaseCW

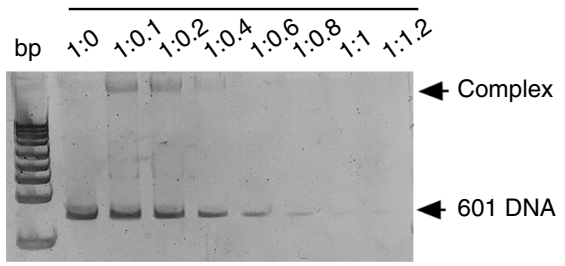

Fig. 5 Mapping the DNA-binding site of the ATPaseCW cassette of MORC4. a A ribbon representation of the dimeric MORC4 ATPaseCW structure. The CW domain is colored yellow, and the ATPase domain is colored green in protomer A and gray in protomer B. The positively charged clusters in ATPaseCW are indicated by ovals and labeled. The residue E56, required for the catalytic activity of ATPaseCW, is also shown in red. b-f EMSA with 601 DNA in the presence of increasing amounts of indicated mutants of MORC4 ATPaseCW.

MORC4 affects the S phase cell cycle. Chromatin organization is dynamic and undergoes extensive changes during the cell cycle, which requires chromatin condensation and decondensation. Because MORC4 enhances the nucleosome stability and homologous MORC1 was found to mediate chromatin compaction ${ }^{25}$, we tested whether MORC4 has a role in regulating the cell cycle.
We assayed the cell cycle state distribution of $\sim 10,000$ HEK293T cells expressing mCherry-tagged wild-type and mutant MORC4 by flow cytometry (Fig. 7c and Supplementary Fig. 13). We found that cells expressing wild-type MORC4 showed a modest, but significantly larger population in S phase of the cell cycle $(38.7 \%)$ compared with the control cells $(34.5 \%)$. These 
a
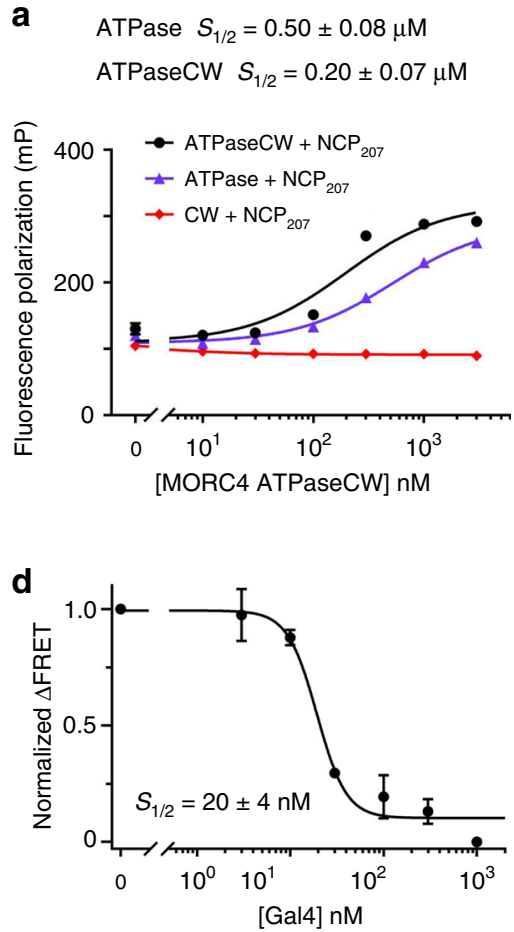

e

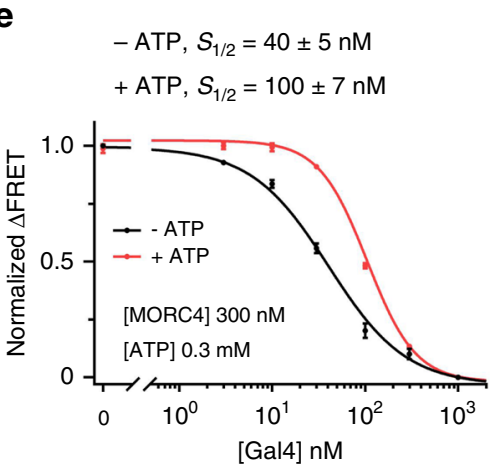

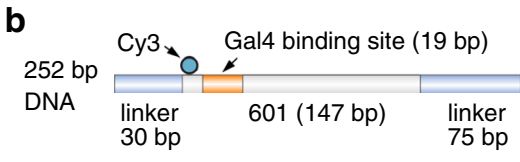
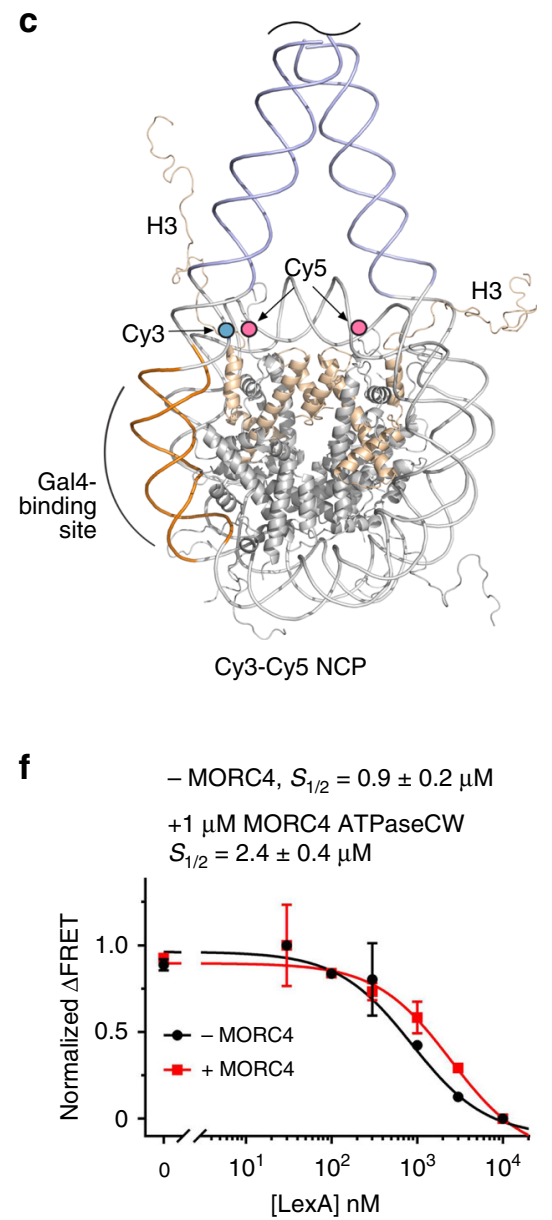

Fig. 6 MORC4 ATPaseCW binds to and stabilizes nucleosomes. a Binding affinities and binding curves for the interactions of the indicated MORC4 regions with $\mathrm{NCP}_{207}$ as measured by fluorescence polarization. Data are represented as mean values +/-S.D. from three independent experiments $(n=3)$. b Schematic of the 252 bp DNA containing the 601 Widom sequence with a 75 bp linker at the 3' end, a 30 bp linker at the $5^{\prime}$ end, and a Gal4 transcription factor binding site at bases 8-26 in the 601 Widom sequence with Cy3 positioned 34 bp from the $5^{\prime}$ end (cyan circle). c A model based on the crystal structure of the NCP with 197 bp palindromic 601 L DNA (PDB ID: 5NLO, the linker histone H1 is not shown) with histone H3 (wheat), Cy5 at H2AK119C (pink circles), Cy3 at the DNA (cyan circle), and the Gal4 target site (orange) are shown and labeled. Truncated DNA linkers are shown and colored light blue. d, e Normalized change in FRET efficiency of the Cy3-Cy5 labeled NCP 252 upon addition of Gal4 in the absence (d) and presence (e) of MORC4 ATPaseCW and +/-ATP. Data are represented as mean values $+/-$ S.D. from three independent experiments $(n=3)$. $\mathbf{f}$ Normalized change in FRET efficiency of the Cy3-Cy5 labeled $\mathrm{NCP}_{147}$ upon the addition of LexA in the absence and presence of MORC4 ATPaseCW. Data are represented as mean values $+/-$ S.D. from three independent experiments $(n=3)$.

results indicate that overexpression of MORC4 alters S phase progression in HEK293T cells. Notably, neither the ATPase mutant (E56A, 36.3\%) nor the CW mutant (W435A, 36.5\%) or the dual mutant $(\mathrm{E} 56 \mathrm{~A} / \mathrm{W} 435 \mathrm{~A}, 35.8 \%)$ altered $\mathrm{S}$ phase progression to the same degree. These data reveal that both ATPase activity and the intact $\mathrm{CW}$ domain are essential (Fig. 7c), but no significant effect was observed for the K460A/R462A/R463A mutant, which suggested that the disruption of the DNA-binding activity of CW is not enough (Supplementary Fig. 13). Our results are consistent with a model whereby MORC4 stabilizes the nucleosome resulting in decreased chromatin accessibility during $S$ phase and slowing replication of DNA. Together, our data point to a role of MORC4 in S-phase progression, which depends on both its catalytic activity and the CW domain.

\section{Discussion}

Among four members of the MORC family of proteins, MORC4 is the least well characterized. In this study we report the molecular and structural mechanism underlying the catalytic ATPase activity of MORC4. Our current model for the MORC4 activation suggests that binding of the ATPase domain and the CW domain of the ATPaseCW cassette to DNA stimulates the catalytic activity of MORC4 and promotes binding of CW to H3K4me3 (Fig. 4b). Notably, our data demonstrate that the DNA-binding function of $\mathrm{CW}$ is required for the MORC4 activation, as the mutant protein with the impaired DNA-binding site of CW loses its ability to be stimulated to hydrolyze ATP.

Despite the overall similar architecture of the MORC proteins, their mechanisms for enzymatic activation differ markedly. In 
a

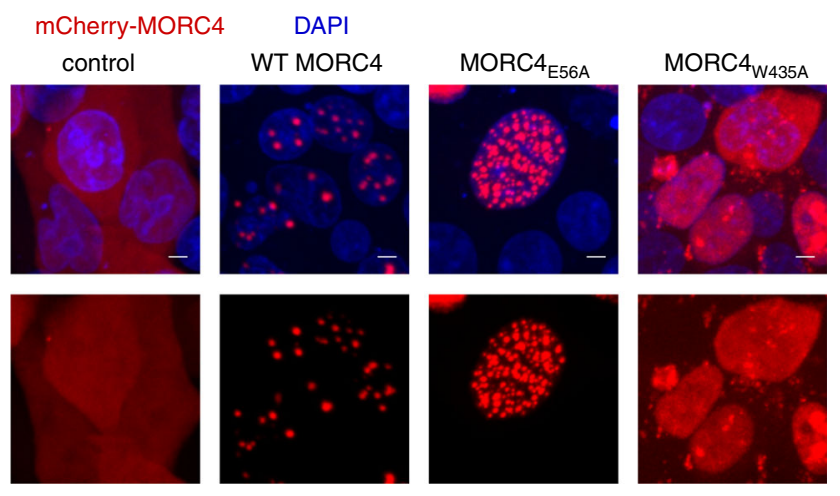

b
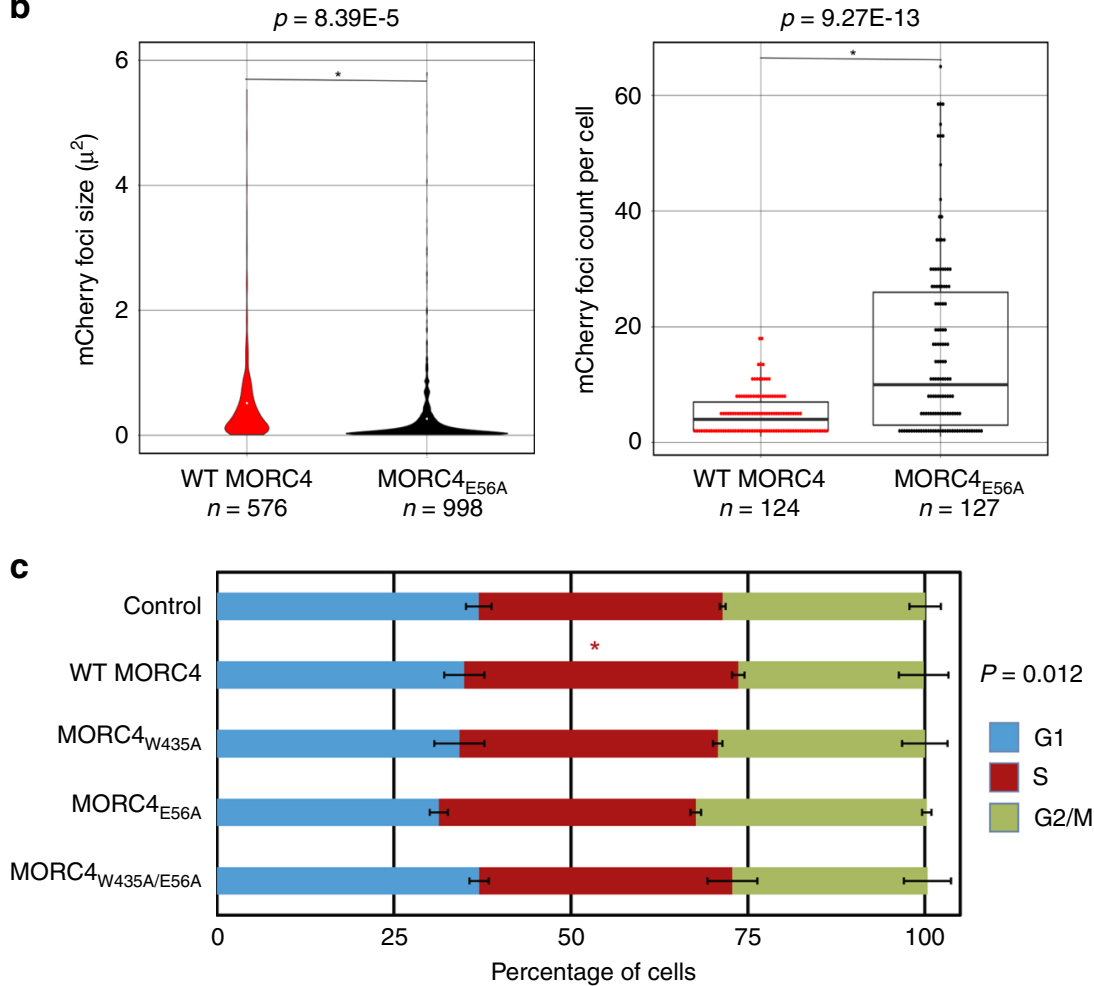

Fig. 7 MORC4 forms NBs and affects the S phase cell cycle. a Representative confocal microscopy images of 293T-HEK cells overexpressing mCherryCTRL, mCherry-MORC4 WT, mCherry-MORC4 E56A or mCherry-MORC4 W435A. Transfection were performed a minimum of three times, scale bars represent $5 \mu \mathrm{m}$. b MORC4 E56A shows a significant decrease in mCherry foci size $(p=8.39 \mathrm{E}-5)$ and a significant increase in number of mCherry foci per cell $(p=9.27 \mathrm{E}-13)$ compared to MORC4 WT. The analysis shown is from at least 10 independent images (WT MORC4 $n=13$, MORC4 E56A $n=15)$, with a minimum of 120 nuclei analyzed for each condition (WT MORC4 $n=124$, MORC4 E56A $n=127$ ). c Cell cycle analysis following 48-h overexpression of MORC4 proteins exhibit increased percent of cells in S phase for WT MORC4 $(p=0.012)$ compared to control cells. Data represent the average of three independent experiments. Error bars represent S.E.M., ${ }^{\star}$ indicates significant difference from mCherry-CTRL $(p<0.05)$ by two-tailed student $t$-test.

contrast to MORC4, the CW domain of MORC3 does not bind DNA and its coupling to the ATPase domain prevents DNA binding by the ATPase domain ${ }^{13,15}$. This results in an autoinhibited, catalytically inactive state of MORC3, which is released through the interaction of $\mathrm{CW}$ with histone $\mathrm{H} 3 \mathrm{~K} 4 \mathrm{me} 3$. In contrast to MORC4 and MORC3, it has been shown that following DNA damage, MORC2 is phosphorylated on Ser739, and this phosphorylation is required for the ATPase activity of MORC2 ${ }^{26}$. The distinctive mechanisms of action of MORCs correlate with the fact that these proteins have unique DNA-binding regions: (i) encompassing the ATPase and CW domains in MORC4, (ii) the ATPase domain only, with the CW domain impeding binding to DNA in MORC $3^{13,15}$, and (iii) a coiled coil (CC) insertion between the ATPase domain and the CW domain in
MORC2 ${ }^{17,27}$. While both DNA binding and dimerization are essential for the ATPase activity of MORC3, mutation of the MORC2 residues that disrupt dimerization interface raises the rate of ATP hydrolysis, and this observation led to a model of MORC2 cycling between monomeric and dimeric states ${ }^{17}$.

Furthermore, MORCs are characterized by different chromatin engaging modes, as their CW domains have dissimilar functions. MORC2 contains a CW domain that is lacking one of the aromatic cage tryptophan residues and thus does not recognize histones. The CW domain of MORC3 is incapable of binding to DNA, and its histone-binding function mediates both the chromatin association and ATPase activity of MORC3. Unlike MORC3 CW, the CW domain of MORC4 is essential in chromatin targeting and binds to histones but it is its DNA-binding 
function that is necessary for the ATPase activity of MORC4. The $\mathrm{CW}$ domain does not inhibit the DNA-binding activity of MORC4 and MORC2, whereas the CW domain of MORC3 does.

Given the stark differences in the mechanisms of action of MORCs, it is expected that each MORC family member may be involved in distinct biological events. Here, we show that in cells, MORC4 plays a role in S-phase progression, possibly by decreasing chromatin accessibility during $\mathrm{S}$ phase and slowing replication of DNA, as in vitro results demonstrate that MORC4 promotes the DNA wrapping in the nucleosome in FRET experiments. While MORC2 is found in the nuclear and cytosolic pools, MORC4 and MORC3 localize exclusively to the nucleus where they form NBs. We have previously shown that MORC3 NBs have LLPS gel-like properties ${ }^{20}$, and it will be essential in future studies to determine the nature of MORC4 NBs. Furthermore, to better understand their roles in chromatin condensation or decondensation, it will be imperative to elucidate the structural modes of MORCs' engagement with the nucleosome.

\section{Methods}

Protein expression and purification. The ATPase domain (aa 29-411) and the ATPaseCW cassette (aa 29-486) of human MORC4 (Q8TE76) were cloned into a pDEST17 vector and pDEST15 vector, respectively. The CW domain (aa 417-475) of MORC4 was cloned into a pGEX 6p-1 vector. Proteins were expressed in Rosetta2 (DE3) pLysS or BL21 (DE3) RIL in LB or minimal media supplemented with ${ }^{15} \mathrm{NH}_{4} \mathrm{Cl}$. Protein expression was induced with $0.2 \mathrm{mM}$ IPTG for $16 \mathrm{~h}$ at $16^{\circ} \mathrm{C}$. The His-tagged ATPase domain was purified on HisPur Ni-NTA agarose beads (Thermo-Fisher) in $20 \mathrm{mM}$ Tris- $\mathrm{HCl}$ (pH 7.5), $300 \mathrm{mM} \mathrm{NaCl}, 5 \mathrm{mM} \mathrm{MgCl}$, and $2 \mathrm{mM}$ DTT. The GST-tagged ATPaseCW cassette was purified on Pierce glutathione agarose beads (Thermo-Fisher) in $20 \mathrm{mM}$ Tris- $\mathrm{HCl}$ (pH 7.5), $300 \mathrm{mM}$ $\mathrm{NaCl}, 5 \mathrm{mM} \mathrm{MgCl}$, and $2 \mathrm{mM}$ DTT. GST-CW was purified on Pierce glutathione agarose beads (Thermo-Fisher) in $20 \mathrm{mM}$ Tris- $\mathrm{HCl}$ (pH 7.5) buffer supplemented with $150 \mathrm{mM} \mathrm{NaCl}$ and $2 \mathrm{mM}$ DTT. The GST tag was cleaved overnight at $4{ }^{\circ} \mathrm{C}$ with either TEV or PreScission proteases. Unlabeled proteins were further purified by size exclusion chromatography and concentrated in Millipore concentrators. All mutants were generated by site-directed mutagenesis using the Stratagene QuikChange mutagenesis protocol, grown and purified as wild-type proteins. MORC3 CW was expressed and purified as reported ${ }^{15}$.

X-ray crystallography. The human MORC4 ATPaseCW cassette (aa 29-486) solution was concentrated to $6 \mathrm{mg} / \mathrm{mL}$ and incubated with the H3K4me3 peptide (aa 1-12 of $\mathrm{H} 3$, in a 1:2 protein-to-peptide molar ratio) along with $2 \mathrm{mM}$ AMPPNP at $25{ }^{\circ} \mathrm{C}$ for $20 \mathrm{~min}$ in buffer containing $20 \mathrm{mM}$ Tris- $\mathrm{HCl}(\mathrm{pH} 7.5), 5 \mathrm{mM} \mathrm{MgCl}_{2}$ and $2 \mathrm{mM}$ DTT. Crystals were obtained at $18^{\circ} \mathrm{C}$ using the sitting-drop vapor diffusion method by mixing equal volumes of protein solution with well solution composed of $0.1 \mathrm{M}$ Hepes (pH 7.5) and 20\% Jeffamine ED-2003. X-ray diffraction data were collected from a single crystal at the ALS 4.2.2 beamline, Berkeley administrated by the Molecular Biology Consortium. HKL2000 was used for indexing, scaling, and data reduction. The phase solution was obtained by molecular replacement using the MORC3 ATPaseCW structure (PDB ID 6O1E) as a search model. Manual model building was performed using $\mathrm{Coot}^{28}$, and the structure was refined using Phenix ${ }^{29}$. The final structure was verified by MOLProbity $^{30}$ and the PDB validation server. The X-ray diffraction and structure refinement statistics are summarized in Supplementary Table 1.

Fluorescence spectroscopy. Spectra were recorded at $25^{\circ} \mathrm{C}$ on a Fluoromax-3 spectrofluorometer (HORIBA). The samples containing $0.5-1 \mu \mathrm{M}$ wild-type or mutated ATPaseCW or CW in $20 \mathrm{mM}$ Tris $\mathrm{pH} 6.8,100 \mathrm{mM} \mathrm{NaCl}, 2 \mathrm{mM}$ DTT buffer and progressively increasing concentrations of the histone peptides were excited at $295 \mathrm{~nm}$. Emission spectra were recorded between 320 and $360 \mathrm{~nm}$ with a $0.5 \mathrm{~nm}$ step size and a $0.5 \mathrm{~s}$ integration time and averaged over three scans. The $K_{\mathrm{d}}$ values were determined using a nonlinear least-squares analysis and the equation:

$$
\Delta I=\Delta I_{\max } \frac{\left(\left([L]+[P]+K_{\mathrm{d}}\right)-\sqrt{\left([L]+[P]+K_{\mathrm{d}}\right)^{2}-4[P][L]}\right)}{2[P]}
$$

where $[L]$ is the concentration of the histone peptide, $[P]$ is the concentration of the protein, $\Delta I$ is the observed change of signal intensity, and $\Delta I_{\max }$ is the difference in signal intensity of the free and bound states of the protein. The $K_{\mathrm{d}}$ values were averaged over three separate experiments with the error calculated as the standard deviation between the runs.

ATPase activity assays. The ATPase assays were performed using the EnzChek Phosphate Assay Kit (Invitrogen, item \# E6646). The reactions were carried out on
$1.0 \mu \mathrm{M}$ of MORC4 His-ATPase or ATPaseCW (WT and mutants), in the presence and absence of $1 \mu \mathrm{M} 601$ DNA or $50 \mu \mathrm{M}$ of unmodified H3 or H3K4me3 peptide (aa 1-12 of H3) in a buffer containing $50 \mathrm{mM}$ Tris- $\mathrm{HCl}$ (pH 7.5), $150 \mathrm{mM} \mathrm{NaCl}$, $1 \mathrm{mM} \mathrm{MgCl}_{2}, 0.1 \mathrm{mM}$ sodium azide, $200 \mu \mathrm{M}$ MESG, and $1 \mathrm{U}$ of PNP. In assay with the nucleosome, $0.5 \mu \mathrm{M}$ of MORC4 ATPaseCW and $0.5 \mu \mathrm{M} \mathrm{NCP} 167$ (Activemotif) were used. The reaction was started by adding $2 \mathrm{mM}$ ATP to the mixture at room temperature, and the release of inorganic phosphate was monitored by measuring the absorbance at $360 \mathrm{~nm}$ on a Nanodrop 2000c spectrophotometer (Thermo Scientific). In the presence of inorganic phosphate, produced by the hydrolysis of ATP to ADP, MESG is enzymatically converted to ribose 1-phosphate and MESG by PNP, resulting in a shift in the wavelength absorbance from $330 \mathrm{~nm}$ for MESG to $360 \mathrm{~nm}$ for the product. The rate of ATP self-hydrolysis was measured in parallel. Error was calculated as the S.D. of at least three separate experiments (two in Fig. 4j).

NMR experiments. NMR experiments were carried out at $298 \mathrm{~K}$ on a Varian INOVA 600 and $900 \mathrm{MHz}$ spectrometers. His-tagged MORC4 ATPase (1-411) was expressed and purified as described above and used without cleavage of the tag. NMR samples contained $0.1 \mathrm{mM}$ uniformly ${ }^{15} \mathrm{~N}$-labeled WT or mutated CW in $20 \mathrm{mM}$ Tris- $\mathrm{HCl}$ (pH 7) buffer supplemented with $100 \mathrm{mM} \mathrm{NaCl}, 5 \mathrm{mM}$ DTT, and $10 \% \mathrm{D}_{2} \mathrm{O}$. Binding was characterized by monitoring CSPs in the proteins induced by $\mathrm{H} 3$ peptides or the ATPase domain. For the $\mathrm{CW}_{\mathrm{KRR}}$ mutant, NMR experiments were performed in $20 \mathrm{mM}$ Tris- $\mathrm{HCl}$ ( $\mathrm{pH}$ 6.8) buffer supplemented with $150 \mathrm{mM}$ $\mathrm{NaCl}, 2 \mathrm{mM}$ DTT, and $10 \% \mathrm{D}_{2} \mathrm{O}$.

Peptide microarrays. Histone peptide microarrays were produced, performed, and analyzed as described ${ }^{31}$ with some modifications. Microarrays were analyzed by the Typhoon Trio+ (GE) and quantified using the ImageQuant TL software. The analysis was performed by first averaging the triplicate values for each peptide in a subarray; these were then linearly scaled based on the minimum and maximum values to be between 0 and 1 . These scaled values were then averaged together and sorted based on the modifications stated in the plot legends. Averages and standard deviations are from $\geq 3$ arrays; these values for every peptide tested can be found in Supplementary Data 1.

Histone peptide pulldown assays. The in-solution histone peptide pulldowns experiments were performed as described ${ }^{31}$. In brief, 50 pmol of MORC4 CW was incubated with 500 pmol of each biotinylated histone peptide in $50 \mathrm{mM}$ Tris, $0.1 \%$ NP-40, $0.5 \% \mathrm{BSA}, 500 \mathrm{mM} \mathrm{NaCl}$, pH 8.0 buffer for one hour at $4{ }^{\circ} \mathrm{C}$ with rotation. Five microliters of magnetic streptavidin-coated beads were added to each reaction and the mixtures were incubated for another hour with rotation. The beads were then washed three times and analyzed using SDS-PAGE and western blotting. Anti-GST (13-0022, 1:1000) antibody was from EpiCypher and anti-Rabbit-HRP (NA934V, 1:20,000) antibody was from GE. The input control lane represents 1\% of the input material used in a pulldown. The images shown are representative of three experiments.

EMSA with DNA. EMSA experiments were performed essentially as described ${ }^{32}$. In brief, increasing amounts of WT or mutant $\mathrm{CW}$ or ATPaseCW cassette of MORC4 were incubated with $147 \mathrm{bp} 601 \mathrm{DNA}$ ( $5 \mathrm{pmol}$ ) or $50 \mathrm{ng}$ of O'RangeRuler 5 bp ladder (Thermo Scientific) in a DNA-binding buffer containing $20 \mathrm{mM}$ Tris$\mathrm{HCl}$ ( $\mathrm{pH} 7.5$ ), $150 \mathrm{mM} \mathrm{NaCl}, 2.5 \mathrm{mM} \mathrm{MgCl}_{2}$, and $2 \mathrm{mM} \mathrm{DTT}$ for $0.5 \mathrm{~h}$ on ice. The reaction mixtures were loaded on $5 \%$ native polyacrylamide gels, and electrophoresis was performed in $0.2 \times$ Tris-borate-EDTA (TBE) buffer at $100 \mathrm{~V}$ for $1.2 \mathrm{~h}$ on ice. Gels were stained with SYBR Gold (Invitrogen).

Cross-linking assay. His-tagged MORC4 (aa 1-486) and MORC4 (29-283, I30A) were expressed and purified as described above. $10 \mu \mathrm{M}$ proteins were cross-linked in $10 \mathrm{mM}$ Hepes pH 7.5, $150 \mathrm{mM} \mathrm{NaCl}, 2 \mathrm{mM} \mathrm{MgCl}, 0.5 \mathrm{mM}$ TCEP, and $1 \mathrm{mM}$ AMPPNP or ADP. BS3 cross-linker (Thermo) was resuspended to $2 \mathrm{mM}$ and used at a concentration of $0.1 \mathrm{mM}$. Cross-linking was allowed to proceed for $10 \mathrm{~min}$. Cross-linked samples were quenched in $0.1 \mathrm{M}$ Tris pH 7.5, resolved on SDS/PAGE, and stained with Coomassie blue.

Fluorescence polarization. Fluorescence polarization measurements were acquired with a Tecan infinite M1000Pro plate reader by exciting at $470 \mathrm{~nm}$ and measuring polarized emission at $519 \mathrm{~nm}$ with $5 \mathrm{~nm}$ excitation and emission bandwidths. The fluorescence polarization was calculated from the emission polarized parallel and perpendicular to the polarized excitation light. Fluorescence polarization measurements were carried out using increasing concentrations of MORC4 ATPaseCW, ATPase alone, or CW alone with $5 \mathrm{nM} 207$ bp Fluoresceinlabeled NCPs in $15 \mathrm{mM}$ Tris pH 7.5, $75 \mathrm{mM} \mathrm{NaCl}, 0.00625 \%$ Tween 20 , and $5 \mathrm{mM}$ dithiothreitol buffer in $30 \mu \mathrm{L}$ reaction volumes. The samples were loaded into a Corning round bottom black polystyrene plate and incubated at room temperature for $10 \mathrm{~min}$ before fluorescence polarization measurements of the samples were taken. The data were then fit to a non-cooperative binding isotherm to determine $S_{1 / 2}$ values. Errors represent a S.D. between the $S_{1 / 2}$ values based on three runs. 
NCP preparation for fluorescence polarization and FRET. Histone octamers unlabeled or labeled with $\mathrm{Cy} 5$ at $\mathrm{H} 2 \mathrm{AK} 119$ were prepared as previously described $^{33}$. NCPs were reconstituted by combining octamer with $1.25 \times$ excess DNA and performing slow salt dialysis. Free DNA was then separated from fully reconstituted NCPs by sucrose gradient purification. NCPs for fluorescence polarization assays were reconstituted from unlabeled octamer and $207 \mathrm{bp}$ DNA containing the 601 Widom sequence flanked with a $30 \mathrm{bp}$ linker on either side and internally labeled with Fluorescein $27 \mathrm{bp}$ in from the $5^{\prime}$ end. Cy3-Cy5 labeled NCPs for FRET assays were reconstituted with Cy5 labeled octamer plus either $147 \mathrm{bp} 601$ Widom DNA containing a LexA transcription factor binding site at bases $8-27^{34}$, or $252 \mathrm{bp}$ DNA containing the 601 Widom sequence with a 75 bp linker at the $3^{\prime}$ end, a $30 \mathrm{bp}$ linker at the 5' end, and a Gal4 transcription factor binding site at bases 8-26 in the 601 Widom sequence. The 147 bp LexA-binding DNA was labeled with Cy3 on the $5^{\prime}$ end, and the $252 \mathrm{bp} \mathrm{Gal4-binding} \mathrm{DNA} \mathrm{was} \mathrm{internally} \mathrm{labeled} \mathrm{with} \mathrm{Cy3} 34 \mathrm{bp}$ in from the $5^{\prime}$ end.

LexA and Gal4 protein preparation. LexA was expressed in E. coli, separated from genomic DNA and the proteome by polyethyleneimine precipitation, and salted out with ammonium sulfate. LexA was resuspended in buffer A $(20 \mathrm{mM}$ potassium phosphate $\mathrm{pH}$ 7, $0.1 \mathrm{mM}$ EDTA, $10 \%$ glycerol, $1 \mathrm{mM} \mathrm{DTT})+200 \mathrm{mM}$ $\mathrm{NaCl}$ and purified by a linear gradient to $\mathrm{A}+800 \mathrm{mM} \mathrm{NaCl}$ over either a cellulose phosphate or HiTrap Heparin HP column. Final LexA purification was performed on a hydroxyapatite column and dialyzed into $10 \mathrm{mM}$ PIPES pH 7.0, $0.1 \mathrm{mM}$ EDTA, $10 \%$ glycerol, and $200 \mathrm{mM} \mathrm{NaCl}$ for storage at $-80{ }^{\circ} \mathrm{C}$. The His-tagged DNA-binding domain (aa 1-147) of Gal4 was expressed in E. coli and purified from lysate by a linear gradient of buffer B $(25 \mathrm{mM}$ Tris pH 7.5, $200 \mathrm{mM} \mathrm{NaCl}$, $0.2 \%$ Tween 20, $20 \mu \mathrm{M}$ zinc acetate, $1 \mathrm{mM}$ dithiothreitol, and $1 \mathrm{mM}$ PMSF) + $10 \mathrm{mM}$ imidazole to B $+200 \mathrm{mM}$ imidazole over a Nickel-NTA column. Gal4 (1-147) was then further purified by a linear gradient of buffer C $(25 \mathrm{mM}$ Tris $\mathrm{pH}$ 7.5, $20 \mu \mathrm{M}$ zinc acetate, $1 \mathrm{mM}$ dithiothreitol, and $1 \mathrm{mM}$ PMSF) $+200 \mathrm{mM} \mathrm{NaCl}$ to $\mathrm{C}+800 \mathrm{mM} \mathrm{NaCl}$ over a Tosoh cation exchange column. Purified Gal4 (1-147) was then dialyzed into $10 \mathrm{mM}$ HEPES pH 7.5, $200 \mathrm{mM} \mathrm{NaCl}, 10 \%$ glycerol, $20 \mu \mathrm{M}$ zinc acetate, $1 \mathrm{mM}$ DTT, and $1 \mathrm{mM}$ PMSF for storage at $-80^{\circ} \mathrm{C}$.

FRET. Cy3-Cy5 NCP FRET efficiency measurements were carried out on a Horiba Scientific Fluoromax 4. Samples were excited at 510 and $610 \mathrm{~nm}$ and the photoluminescence spectra were collected from 530 to $750 \mathrm{~nm}$ and 630 to $750 \mathrm{~nm}$ for donor and acceptor excitations, respectively. Each wavelength was integrated for one second, and the excitation and emission slit width were set to $5 \mathrm{~nm}$ with $2 \mathrm{~nm}$ emission wavelength steps. FRET measurements were computed through the (ratio)A method. LexA titrations were carried out in $20 \mathrm{mM}$ Tris pH 7.5, $75 \mathrm{mM}$ $\mathrm{NaCl}, 10 \%$ glycerol, $0.00625 \%$ Tween 20 buffer with $5 \mathrm{nM} 147$ bp Cy3-Cy5 NCPs mixed in $20 \mu \mathrm{L}$ reaction volumes and allowed to incubate for 5 mins at $4{ }^{\circ} \mathrm{C}$ before FRET of the sample was measured. To measure changes in accessibility of the $\mathrm{NCP}_{147}$ due to MORC4 ATPaseCW binding, LexA titrations were repeated in the presence of NCP binding saturating concentrations of MORC4 ATPaseCW. Gal4 (1-147) titrations were carried out in $10 \mathrm{mM}$ Tris $\mathrm{pH} 8,130 \mathrm{mM} \mathrm{NaCl}, 10 \%$ glycerol, $0.0075 \%$ Tween 20 buffer with $1 \mathrm{nM} 252$ bp Cy3-Cy5 nucleosomes mixed in $60 \mu \mathrm{L}$ reaction volumes and allowed to incubate for 10 mins at room temperature before FRET of the sample was measured. To measure changes in accessibility of the $\mathrm{NCP}_{252}$ due to MORC4 ATPaseCW binding, Gal4 (1-147) titrations were repeated in the presence of NCP binding saturating concentrations of MORC4 ATPaseCW $+/-0.3 \mathrm{mM}$ ATP. FRET efficiency values were normalized against the FRET efficiency value of NCP in the absence of titrant. The titrations were then fit to a non-cooperative binding isotherm to determine $S_{1 / 2}$ values. Errors represent a S.D. between the $S_{1 / 2}$ values based on three runs.

EMSA with NCPs. EMSAs were performed by mixing increasing amounts of MORC4 ATPase with $5 \mathrm{nM} 147 \mathrm{bp}$ fluorescein-labeled NCP in $25 \mathrm{mM}$ Tris-HCl pH 7.5, $75 \mathrm{mM} \mathrm{NaCl}, 25 \mathrm{mM}$ Tris- $\mathrm{HCl} \mathrm{pH} \mathrm{7.5,} \mathrm{0.005 \%} \mathrm{Tween} \mathrm{20,} \mathrm{and} \mathrm{10 \%} \mathrm{glycerol}$ buffer in a $12 \mu \mathrm{L}$ reaction volume. Each sample was incubated at $4{ }^{\circ} \mathrm{C}$ for $5 \mathrm{~min}$ and then loaded onto a $5 \%$ native polyacrylamide gel. Electrophoresis was performed in $0.3 \times$ Tris-borate-EDTA (TBE) at $300 \mathrm{~V}$ for $90 \mathrm{~min}$. Fluorescein fluorescence images were acquired with a Typhoon Phosphor Imager.

Cell culture. 293T-HEK cells were maintained in Dulbecco's Modified Essential Medium (DMEM) with 10\% FB Essence (VWR), 1\% penicillin/streptomycin and L-glutamine.

MORC4 localization in HEK cells. WT mCherry-MORC4, mCherry-MORC4 E56A, mCherry-MORC4 W435A, mCherry-MORC4 E56A/W435A, mCherryMORC4 K460A/R462A/R463A, or mCherry control plasmid were transfected into 2 million 293T-HEK cells seeded on $25 \mathrm{~mm}$ circular coverslips (Carolina Biological Supply Item\#: 633057) in 10-cm-diameter tissue culture dish by Lipofectamine 3000 (Life Technology, L3000-075) following the manufacturer's instructions. Two days after transfection, cells were stained with Hoechst 333241 for $30 \mathrm{~min}$ and imaged live on a ZeissAxiovert $200 \mathrm{M}$ inverted microscope fitted with a $\times 40$ oil objective. Images were acquired with Slidebook 6 software and exported for analysis in Image J.
mCherry foci size and foci per cell calculations. mCherry foci sizes were calculated using ImageJ image processing program (version 1.51) using the Analyze Particle tool. mCherry particle sizes were collected for MORC4 WT and MORC4 W435A transfected cells. Data were plotted with R (version 3.4.1) using ggplot2 (version 3.2.1). Cell size and mCherry foci count per cell were calculated using CellProfile cell image analysis software (version 3.1.5) to identify nuclei and mCherry foci, relate mCherry foci to nuclei containing them and measure the size of nuclei and number mCherry foci contained. Data were plotted with $\mathrm{R}$ (version 3.4.1) using ggplot2 (version 3.2.1).

Flow cytometry. For DNA content analysis, $10^{6}$ cells previously transfected with mCherry MORC4 constructs and labeled with $25 \mu \mathrm{M}$ Ethynyl deoxyUridine (EdU) for $1 \mathrm{~h}$ in $100 \mu \mathrm{L}$ were washed with PBS $\left(\mathrm{NaCl} 137 \mathrm{mM}, \mathrm{KCl} 2.7 \mathrm{mM}, \mathrm{Na}_{2} \mathrm{HPO}_{4}\right.$ $10 \mathrm{mM}, \mathrm{KH}_{2} \mathrm{PO}_{4} 1.8 \mathrm{mM}$ ) and fixed in ice cold $70 \%$ ethanol overnight. Fixed cells were washed and resuspended in PBS containing $0.05 \%$ NP40 and incubated at room temperature for $30 \mathrm{~min}$. Cells were again washed with PBS. Cells were resuspended in Alexa Fluor 647 click staining solution (100 mM Tris pH 7.6, $4 \mathrm{mM}$ $\mathrm{CuSO}_{4}, 3 \mu \mathrm{M}$ AF647 azide, $100 \mathrm{mM}$ Sodium Ascorbate) for $1 \mathrm{~h}$. Following staining, cells were washed with PBS and then stained for total DNA content using $10 \mu \mathrm{g} / \mathrm{mL}$ propidium iodide (PI) and $0.2 \mathrm{mg} / \mathrm{ml}$ RNase A for $60 \mathrm{~min}$ before analysis on a FACScan instrument. Data were analyzed using FlowJo (Version 10) with cells gated to remove doublets. EdU vs PI plots were gated in quadrants to identify G1, early S, late $\mathrm{S}$ and G2/M cells. S phase fractions were summed for total S phase population. Four biological replicated were conducted and the average percentages for each phase were plotted.

Reporting summary. Further information on research design is available in the Nature Research Reporting Summary linked to this article.

\section{Data availability}

Coordinates and structure factors for the ATPaseCW cassette have been deposited in the Protein Data Bank (PDB 7K7T). All relevant data supporting the key findings of this study are available within the article and its Supplementary Information files or from the corresponding author upon reasonable request. Source data are provided with this paper.

Received: 17 February 2020; Accepted: 7 October 2020;

Published online: 29 October 2020

\section{References}

1. Angsuwatcharakon, P., Sodsai, P., Rerknimitr, R. \& Hirankarn, N. The Polymorphisms at PRSS1-PRSS2 and MORC4 loci and the risk of Post-ERCP pancreatitis. Gastroenterol. Res, Pr. 2018, 1064783 (2018).

2. Derikx, M. H. et al. Polymorphisms at PRSS1-PRSS2 and CLDN2-MORC4 loci associate with alcoholic and non-alcoholic chronic pancreatitis in a European replication study. Gut 64, 1426-1433 (2015).

3. Duan, X. et al. Baicalin inhibits cell viability, migration and invasion in breast cancer by regulating miR-338-3p and MORC4. OncoTargets Ther. 12, 11183-11193 (2019)

4. Hong, G. et al. The emerging role of MORC family proteins in cancer development and bone homeostasis. J. Cell Physiol. 232, 928-934 (2017).

5. Liggins, A. P. et al. MORC4, a novel member of the MORC family, is highly expressed in a subset of diffuse large B-cell lymphomas. Br. J. Haematol. 138, 479-486 (2007).

6. Paliwal, S. et al. Association analysis of PRSS1-PRSS2 and CLDN2-MORC4 variants in nonalcoholic chronic pancreatitis using tropical calcific pancreatitis as model. Pancreas 45, 1153-1157 (2016).

7. Soderman, J. et al. Analysis of single nucleotide polymorphisms in the region of CLDN2-MORC4 in relation to inflammatory bowel disease. World J. Gastroenterol. 19, 4935-4943 (2013).

8. Weiss, F. U. et al. Common variants in the CLDN2-MORC4 and PRSS1PRSS2 loci confer susceptibility to acute pancreatitis. Pancreatology 18 477-481 (2018)

9. Yang, Z., Zhuang, Q., Hu, G. \& Geng, S. MORC4 is a novel breast cancer oncogene regulated by miR-193b-3p. J. Cell Biochem. 120, 4634-4643 (2019)

10. Li, D. Q., Nair, S. S. \& Kumar, R. The MORC family: new epigenetic regulators of transcription and DNA damage response. Epigenetics 8, 685-693 (2013).

11. Dutta, R. \& Inouye, M. GHKL, an emergent ATPase/kinase superfamily. Trends Biochem. Sci. 25, 24-28 (2000).

12. Li, X. et al. Quantitative chemical proteomics approach to identify posttranslational modification-mediated protein-protein interactions. J. Am. Chem. Soc. 134, 1982-1985 (2012).

13. Andrews, F. H. et al. Multivalent chromatin engagement and inter-domain crosstalk regulate MORC3 ATPase. Cell Rep. 16, 3195-3207 (2016). 
14. Li, S. et al. Mouse MORC3 is a GHKL ATPase that localizes to H3K4me3 marked chromatin. Proc. Natl Acad. Sci. USA 113, E5108-E5116 (2016).

15. Zhang, Y. et al. Mechanism for autoinhibition and activation of the MORC3 ATPase. Proc. Natl Acad. Sci. USA https://doi.org/10.1073/pnas.1819524116 (2019).

16. Liu, Y. et al. Family-wide characterization of histone binding abilities of human CW domain-containing proteins. J. Biol. Chem. 291, 9000-9013 (2016).

17. Douse, C. H. et al. Neuropathic MORC2 mutations perturb GHKL ATPase dimerization dynamics and epigenetic silencing by multiple structural mechanisms. Nat. Commun. 9, 651 (2018)

18. Tchasovnikarova, I. A. et al. Hyperactivation of HUSH complex function by Charcot-Marie-Tooth disease mutation in MORC2. Nat. Genet. 49, 1035-1044 (2017).

19. Zhang, Y. et al. MORC3 is a target of the influenza A viral protein NS1. Structure 27, 1029-1033.e3 (2019).

20. Zhang, Y. et al. MORC3 forms nuclear condensates through phase separation. iScience 17, 182-189 (2019).

21. Musselman, C. A., Lalonde, M. E., Cote, J. \& Kutateladze, T. G. Perceiving the epigenetic landscape through histone readers. Nat. Struct. Mol. Biol. 19, 1218-1227 (2012).

22. Andrews, F. H., Strahl, B. D. \& Kutateladze, T. G. Insights into newly discovered marks and readers of epigenetic information. Nat. Chem. Biol. 12, 662-668 (2016).

23. Li, G. \& Widom, J. Nucleosomes facilitate their own invasion. Nat. Struct. Mol. Biol. 11, 763-769 (2004).

24. Luo, Y., North, J. A., Rose, S. D. \& Poirier, M. G. Nucleosomes accelerate transcription factor dissociation. Nucleic Acids Res. 42, 3017-3027 (2014).

25. Kim, H. et al. The gene-silencing protein MORC-1 topologically entraps DNA and forms multimeric assemblies to cause DNA compaction. Mol. Cell 75, 700-710 e6 (2019).

26. Li, D. Q. et al. MORC2 signaling integrates phosphorylation-dependent, ATPase-coupled chromatin remodeling during the DNA damage response. Cell Rep. 2, 1657-1669 (2012).

27. Liu, Y. et al. Family-wide characterization of histone binding abilities of human CW domain containing proteins. J. Biol. Chem. 291, 9000-9013 (2016).

28. Emsley, P., Lohkamp, B., Scott, W. G. \& Cowtan, K. Features and development of Coot. Acta Crystallogr. D. Biol. Crystallogr. 66, 486-501 (2010).

29. Adams, P. D. et al. PHENIX: a comprehensive Python-based system for macromolecular structure solution. Acta Crystallogr. D. Biol. Crystallogr. 66, 213-221 (2010).

30. Chen, V. B. et al. MolProbity: all-atom structure validation for macromolecular crystallography. Acta Crystallogr. D. Biol. Crystallogr. 66 12-21 (2010)

31. Petell, C. J., Pham, A. T., Skela, J. \& Strahl, B. D. Improved methods for the detection of histone interactions with peptide microarrays. Sci. Rep. 9, 6265 (2019).

32. Klein, B. J. et al. Bivalent interaction of the PZP domain of BRPF1 with the nucleosome impacts chromatin dynamics and acetylation. Nucleic Acids Res. 44, 472-484 (2016).

33. Tencer, A. H. et al. Covalent modifications of histone H3K9 promote binding of CHD3. Cell Rep. 21, 455-466 (2017).
34. Gibson, M. D., Gatchalian, J., Slater, A., Kutateladze, T. G. \& Poirier, M. G. PHF1 Tudor and N-terminal domains synergistically target partially unwrapped nucleosomes to increase DNA accessibility. Nucleic Acids Res. 45, 3767-3776 (2017)

\section{Acknowledgements}

We thank JaeWoo Ahn for help with experiments. This work was supported by grants from NIH GM125195, HL151334 and GM135671 to T.G.K., GM121966 and GM120582 to M.G.P., GM128720 to J.C.B and GM126900 to B.D.S. A.H.T. is an American Heart Association postdoctoral fellow. Y.Z. is supported by NIH K99CA241301. C.J.P. is supported by the NIH grant T32AA007464 and is an American Cancer Society postdoctoral fellow.

\section{Author contributions}

A.H.T,, K.L.C., G.M.W., Y.Z., C.J.P., and B.J.K., performed experiments and together with B.D.S., J.C.B., M.G.P., and T.G.K. analyzed the data. T.G.K. wrote the manuscript with input from all authors.

\section{Competing interests}

The authors declare no competing interests.

\section{Additional information}

Supplementary information is available for this paper at https://doi.org/10.1038/s41467 020-19278-8.

Correspondence and requests for materials should be addressed to T.G.K.

Peer review information Nature Communications thanks Pablo De Ioannes, and the other, anonymous, reviewer(s) for their contribution to the peer review of this work. Peer reviewer reports are available.

Reprints and permission information is available at http://www.nature.com/reprints

Publisher's note Springer Nature remains neutral with regard to jurisdictional claims in published maps and institutional affiliations.

(c) Open Access This article is licensed under a Creative Commons Attribution 4.0 International License, which permits use, sharing, adaptation, distribution and reproduction in any medium or format, as long as you give appropriate credit to the original author(s) and the source, provide a link to the Creative Commons license, and indicate if changes were made. The images or other third party material in this article are included in the article's Creative Commons license, unless indicated otherwise in a credit line to the material. If material is not included in the article's Creative Commons license and your intended use is not permitted by statutory regulation or exceeds the permitted use, you will need to obtain permission directly from the copyright holder. To view a copy of this license, visit http://creativecommons.org/ licenses/by/4.0/.

(C) The Author(s) 2020 